\title{
The Spherical Tensor Gradient Operator
}

\author{
Ernst Joachim Weniger \\ Institut für Physikalische und Theoretische Chemie \\ Universität Regensburg, D-93040 Regensburg, Germany \\ joachim.weniger@chemie.uni-regensburg.de \\ To Appear in the Josef Paldus Birthday lssue of \\ Collection of Czechoslovak Chemical Communications \\ Date of Submision: 3 February 2005 \\ Date of Acceptance: 15 April 2005 \\ Final Submission: 6 May 2005
}

Dedicated to Professor Josef Paldus on the occasion of his 70th
birthday

PACS numbers: 03.65-w, 03.65.Fd, 02.30.Gp

\begin{abstract}
The spherical tensor gradient operator $\mathcal{Y}_{\ell}^{m}(\nabla)$, which is obtained by replacing the Cartesian components of $\boldsymbol{r}$ by the Cartesian components of $\nabla$ in the regular solid harmonic $\mathcal{Y}_{\ell}^{m}(\boldsymbol{r})$, is an irreducible spherical tensor of rank $\ell$. Accordingly, its application to a scalar function produces an irreducible spherical tensor of rank $\ell$. Thus, it is in principle sufficient to consider only multicenter integrals of scalar functions: Higher angular momentum states can be generated by differentiation with respect to the nuclear coordinates. Many of the properties of $\mathcal{Y}_{\ell}^{m}(\nabla)$ can be understood easily with the help of an old theorem on differentiation by Hobson [Proc. London Math. Soc. 24, 54 - 67 (1892)]. It follows from Hobson's theorem that some scalar functions of considerable relevance as for example the Coulomb potential, Gaussian functions, or reduced Bessel functions produce particularly compact results if $\mathcal{Y}_{\ell}^{m}(\nabla)$ is applied to them. Fourier transformation is very helpful to understand the properties of $\mathcal{Y}_{\ell}^{m}(\nabla)$ since it produces $\mathcal{Y}_{\ell}^{m}(-\mathrm{i} \boldsymbol{p})$. It is also possible to apply $\mathcal{Y}_{\ell}^{m}(\nabla)$ to generalized functions, yielding for instance the
\end{abstract}


spherical delta function $\delta_{\ell}^{m}(\boldsymbol{r})$. The differential operator $\mathcal{Y}_{\ell}^{m}(\nabla)$ can also be used for the derivation of pointwise convergent addition theorems. The feasibility of this approach is demonstrated by deriving the addition theorem of $r^{\nu} \mathcal{Y}_{\ell}^{m}(\boldsymbol{r})$ with $\nu \in \mathbb{R}$.

\section{Contents}

1 Introduction 3

\begin{tabular}{|lll}
2 & The Spherical Tensor Gradient Operator & 8
\end{tabular}

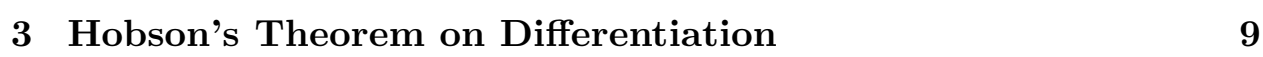

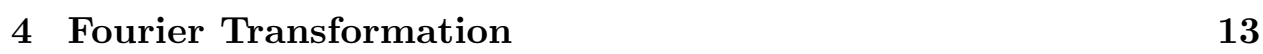

$\begin{array}{lll}5 & \text { Reduced Bessel Functions } & 19\end{array}$

6 Spherical Delta Functions 23

$\begin{array}{lll}7 & \text { Addition Theorems } & 29\end{array}$

$\begin{array}{lll}8 & \text { Summary and Conclusions } & 37\end{array}$

A Appendix: Terminology and Definitions 41

B Appendix: Spherical Harmonics 41

C Appendix: Gaunt Coefficients 43

Ernst Joachim Weniger: The Spherical Tensor Gradient Operator 


\section{Introduction}

In the early 17th century, scientific methodology advanced significantly by what Kline [56, Chapter 16] called the mathematization of science. Roughly at the same time, the foundations for the later development of calculus were laid (see for example [56. Chapter 17]). Differential and integral calculus greatly extended the arsenal of mathematical techniques that could be used for a description and analysis of scientific phenomena. Ultimately, this development led to a period of unity between mathematics and sciences which lasted approximately to the end of the 19th or to the beginning of the 20th century. In that period, it was frequently not possible to decide whether somebody was predominantly a mathematician or predominantly a scientist, and mathematics and the sciences enriched each other greatly by cross fertilization.

A striking example for this unity between mathematics and the sciences is provided by Peter Debye who had been a student of Sommerfeld. Among chemists, Debye is best known for his work on electrolyte solutions, which earned him the Nobel Prize in Chemistry in 1936. It is, however, not nearly so well known that he was also an excellent mathematician who made significant contribution to the theory of Bessel and Hankel functions (see for example [115, Chapter VIII]).

Since the times of Debye, the amount of collective knowledge has increased tremendously. Accordingly, contemporary research is predominantly done by specialists, who know almost everything about almost nothing, whereas generalists like Debye, who had done excellent research in mathematics, physics, and physical chemistry, have become exceedingly rare. In particular, there is a widening gap between mathematicians and those that apply mathematics. In the past, mathematicians had developed new analytical or numerical techniques that greatly helped scientists and engineers to do research in their disciplines. In return, open scientific or engineering problems had always provided a valuable source of inspiration for mathematicians. Unfortunately, this is now longer true. Due to increased specialization, communication between mathematics and those disciplines, that use mathematics as their main language, has deteriorated. This is certainly a deplorable development because cross fertilizations typically happens at the interfaces of different disciplines.

Fortunately, encouraging counterexamples still exist: For example, Professor Josef Paldus started his career at the Heyrovský Institute in Prague

Ernst Joachim Weniger: The Spherical Tensor Gradient Operator 
doing experimental and theoretical work in electrochemistry, and he later also worked as an experimental spectroscopist. In addition, he soon ventured into the newly emerging field of quantum chemistry. In 1968, he came as Brezhnev's gift to the Department of Applied Mathematics of the University of Waterloo. There, his predominant research interest has been the treatment of electronic correlation, emphasizing highly sophisticated mathematical techniques as for example the representation theory of Lie groups. It was this strong mathematical orientation of his research which earned him - in addition to numerous other honors - the Fellowship of the prestigious Fields Institute for Research in Mathematical Sciences.

Quite in the spirit of the highly interdisciplinary research of Professor Josef Paldus, I want to discuss in this article a topic that is located somewhere at the interface between mathematics and atomic and molecular electronic structure theory.

Since space is isotropic, free atoms are spherically symmetric and angular momentum is conserved. Thus, spherical polar coordinates and the machinery of angular momentum theory lead to significant computational and conceptual simplifications in atomic structure calculations. It is probably only a slight exaggeration to claim that atomic electronic structure theory is essentially an application of angular momentum theory.

In the case of molecules, the benefits of spherical coordinates and angular momentum theory are not so obvious. In general, molecules possess no spatial symmetry at all, and if they have one, it is a lower symmetry than spherical symmetry. Thus, neither spherical polar coordinates nor angular momentum theory lead to a such spectacular reduction of computational complexity as they do in the case of atoms.

However, chemists have found it helpful to approach molecular electronic structure theory from the perspective of atoms. For example, the most successful computational scheme, the so-called LCAO-MO approach, is based on the tacit assumption that the parentage of atoms facilitates our attempts of understanding the electronic structure of molecules. Thus, in molecular electronic structure calculations it is common to use angular momentum theory at least locally, i.e., with respect to the atomic centers. In this way, at least some of the formal advantages of angular momentum theory can be retained.

Nevertheless, the use of angular momentum theory for systems lacking rotational symmetry causes new mathematical and computational challenges. In the case of atoms, only relative small angular momentum quantum num-

Ernst Joachim Weniger: The Spherical Tensor Gradient Operator 
bers occur, and the coupling of irreducible spherical tensors produces finite expressions consisting of a few terms only. In the case of molecules, the situation is in general much more complicated: We have to deal with infinite series expansions over angular momentum states that do not necessarily converge rapidly. Thus, we must be able to compute the typical quantities of angular momentum theory both efficiently and reliably even for very large angular momentum quantum numbers. Quite often, this is not so easy.

In molecular electronic structure calculations with analytic basis functions centered at the atomic nuclei, the basic quantities are matrix elements, i.e., essentially multicenter integrals whose evaluation may be very difficult. It is an undeniable empirical fact that it is often comparatively easy to obtain explicit analytical expressions for multicenter integrals over functions that are scalars or irreducible spherical tensors of rank zero with respect to their local (atomic) coordinate systems. If, however, the functions occurring in the multicenter integral are irreducible spherical tensors of higher ranks, one can easily get lost in an algebraic jungle and the derivation of explicit expressions can become extremely difficult or even impossible.

It is another empirical fact that it is usually much easier to differentiate than to integrate. Accordingly, it is an obvious idea to try to generate an explicit expression for a multicenter integral over nonscalar functions by differentiating the simpler expression for the corresponding integral over scalar functions (preferably the simplest scalar functions) with respect to scaling parameters and/or nuclear coordinates (compare also [129, Section IV]). The use of generating differential operators does not necessarily produce closed form expression that hold for arbitrary quantum numbers. Instead, it may be necessary to derive a new expression for each set of quantum numbers. Obviously, a multitude of special formulas is not nearly as convenient as a neat general explicit expression, but powerful computer algebra systems like Maple or Mathematica with the ability of automatically generating FORTRAN or C code help to make such an approach feasible (see for example [15]).

It is relatively easy to generate multicenter integrals of higher scalar functions by differentiating the most simple scalar functions with respect to their scaling parameters. In the case of a $1 s$ Slater-type or Gaussian function, we can construct higher scalar functions easily by repeatedly using the relationships $\partial \exp (-\alpha r) / \partial \alpha=-r \exp (-\alpha r)$ or $\partial \exp \left(-\alpha r^{2}\right) / \partial \alpha=-r^{2} \exp (-\alpha r)$.

The generation of anisotropic functions, that are irreducible spherical tensors of rank $\ell$, from scalar functions is less straightforward: It follows

Ernst Joachim Weniger: The Spherical Tensor Gradient Operator 
from Hobson's theorem [42, which is discussed in Section 3, that we have to apply the spherical tensor gradient operator $\mathcal{Y}_{\ell}^{m}(\nabla)$ to scalar functions. This differential operator is obtained by replacing in the regular solid harmonic $\mathcal{Y}_{\ell}^{m}(\boldsymbol{r})=r^{\ell} Y_{\ell}^{m}(\theta, \phi)$ the Cartesian components of $\boldsymbol{r}=(x, y, z)$ by the Cartesian components of $\nabla=(\partial / \partial x, \partial / \partial y, \partial / \partial z)$.

I came across the differential operator $\mathcal{Y}_{\ell}^{m}(\nabla)$ during my $\mathrm{PhD}$ thesis 117 . in which I worked on multicenter integrals of reduced Bessel functions and their anisotropic generalizations, the so-called $B$ functions. These functions, which are discussed in Section 5 , are a special class of exponentially decaying functions with some very useful properties. When I derived the remarkably compact Fourier transform (5.6) of a $B$ function [117, Eq. (7.1-6) on p. 160], I noticed that the Fourier integral representation (5.8) of an anisotropic $B$ function $B_{n, \ell}^{m}(\alpha, \boldsymbol{r})$ differs from that of the scalar $B$ function $B_{n+\ell, 0}^{m}(\alpha, \boldsymbol{r})$ only by an additional regular solid harmonic $\mathcal{Y}_{\ell}^{m}(-\mathrm{i} \boldsymbol{p})$. Because of (4.4), $\mathcal{Y}_{\ell}^{m}(\mathrm{i} \boldsymbol{p})$ can be interpreted to be the Fourier transform of the differential operator $\mathcal{Y}_{\ell}^{m}(\nabla)$, and I immediately deduced that the anisotropic $B$ function $B_{n, \ell}^{m}(\alpha, \boldsymbol{r})$ can according to (5.9) be generated by applying $\mathcal{Y}_{\ell}^{m}(\nabla)$ to the scalar $B$ function $B_{n+\ell, 0}^{m}(\alpha, \boldsymbol{r})$ [117, Eq. (7.1-10) on p. 161].

Of course, this observation aroused my interest: I wanted to know whether this result can also be proved directly without the help of Fourier transformation, and I also wanted to know whether the differential operator $\mathcal{Y}_{\ell}^{m}(\nabla)$ is a useful analytical tool in different contexts. After the completion of my $\mathrm{PhD}$ thesis, I studied the spherical tensor gradient operator more seriously. I soon learned that I was not the only one and in particular not the first one who had studied and used this differential operator: The first article dealing with the spherical tensor gradient operator, which I am aware of, was published by Hobson [42] in 1892. I also noticed that $\mathcal{Y}_{\ell}^{m}(\nabla)$ is a highly useful analytical tool for a wide range of problems. For example, I found recent articles which describe successful applications of $\mathcal{Y}_{\ell}^{m}(\nabla)$ in scattering [14] or in solid state theory [13, 69].

From the perspective of quantum chemistry, it is probably more interesting that $\mathcal{Y}_{\ell}^{m}(\nabla)$ can be extremely useful in the context of molecular multicenter integrals of exponentially decaying functions, as shown in articles by Grotendorst and Steinborn [39, 40, Niukkanen [70, 71, 73, Novosadov 75, 76, 77, 78, 79, 80, Tai [113, and in my own research [109, 120, 122, 126, 129, 131, 133, 136.

The spherical tensor gradient operator $\mathcal{Y}_{\ell}^{m}(\nabla)$ is - as discussed in more details in Section 3- also extremely useful in the context of multicenter in-

Ernst Joachim Weniger: The Spherical Tensor Gradient Operator 
tegrals of spherical Gaussian functions. For example, Dunlap reformulated in a series of recent articles [22, 23, 24, 25, 26] the theory of multicenter integrals of anisotropic spherical Gaussians by systematically applying $\mathcal{Y}_{\ell}^{m}(\nabla)$ to the corresponding integrals of $1 s$ Gaussians. Many more references on multicenter integrals of spherical Gaussians can be found in Section 3 ,

These examples should suffice to show that $\mathcal{Y}_{\ell}^{m}(\nabla)$ is indeed a very useful analytical tool that has been applied successfully to a wide range of problems. Nevertheless, a reasonably comprehensive discussion the mathematical properties of the spherical tensor gradient operator from the perspective of a potential future user seems to be missing. This is what I intend to provide with this article.

In Section 2] the spherical tensor gradient operator is introduced and some general features - in particular those based on its tensorial nature are discussed.

Section 3 treats Hobson's theorem on differentiation, by means of which many properties of $\mathcal{Y}_{\ell}^{m}(\nabla)$ can be derived and understood easily.

Fourier transformation, which is discussed in Section 4 maps the gradient operator $\nabla$ to i $\boldsymbol{p}$, or equivalently $\mathcal{Y}_{\ell}^{m}(\nabla)$ to $\mathcal{Y}_{\ell}^{m}(\mathrm{i} \boldsymbol{p})$. Thus, in momentum space, we only have to study the comparatively simple multiplicative operator $\mathcal{Y}_{\ell}^{m}(\mathrm{i} \boldsymbol{p})$ whose properties are very well understood. This greatly facilitates a theoretical analysis.

In Section [5, the so-called reduced Bessel functions and their anisotropic generalizations, the so-called $B$ functions, are discussed. These functions play a special role among exponentially decaying functions because of their exceptionally simple Fourier transform and because of the ease, with which the spherical tensor gradient operator can be applied.

Classically, the domain of the spherical tensor gradient operator consists of the differentiable functions $f: \mathbb{R}^{3} \rightarrow \mathbb{C}$, but it makes sense to apply it also to generalized functions. Thus, in Section 6 the spherical delta function and related objects - for example distributional $B$ functions - are treated.

In Section 7 the derivation of addition theorems of essentially arbitrary irreducible spherical tensors with the help of the spherical tensor gradient operator is discussed. In addition, the addition theorem of $r^{\nu} \mathcal{Y}_{\ell}^{m}(\boldsymbol{r})$ with $\nu \in \mathbb{R}$ is constructed in order to demonstrate the feasibility of the whole approach.

This article is concluded by a short summary in Section 8 Finally, there are three Appendices: The terminology used in this article is introduced in Appendix A the for our purposes most relevant properties of the spherical

Ernst Joachim Weniger: The Spherical Tensor Gradient Operator 
harmonics are reviewed in Appendix [B, and Gaunt coefficients are discussed in Appendix C.

\section{The Spherical Tensor Gradient Operator}

The spherical tensor gradient operator $\mathcal{Y}_{\ell}^{m}(\nabla)$ can be introduced via the explicit expression (B.12) for the regular solid harmonic $\mathcal{Y}_{\ell}^{m}(\boldsymbol{r})$. This explicit expression holds for the Cartesian components of essentially arbitrary threedimensional vectors. Thus, we obtain the differential operator $\mathcal{Y}_{\ell}^{m}(\nabla)$ if we replace in (B.8) the Cartesian components of $\boldsymbol{r}=(x, y, z)$ by the Cartesian components of $\nabla=(\partial / \partial x, \partial / \partial y, \partial / \partial z)$ :

$$
\begin{aligned}
\mathcal{Y}_{\ell}^{m}(\nabla)= & {\left[\frac{2 \ell+1}{4 \pi}(\ell+m) !(\ell-m) !\right]^{1 / 2} } \\
& \times \sum_{k \geq 0} \frac{\left(-\frac{\partial}{\partial x}-\mathrm{i} \frac{\partial}{\partial y}\right)^{m+k}\left(\frac{\partial}{\partial x}-\mathrm{i} \frac{\partial}{\partial y}\right)^{k}\left(\frac{\partial}{\partial z}\right)^{\ell-m-2 k}}{2^{m+2 k}(m+k) ! k !(\ell-m-2 k) !} .
\end{aligned}
$$

A new differential operators is not necessarily a useful thing, let alone a major achievement. In atomic and molecular calculations, we are interested in functions that are in defined in terms of spherical polar coordinates and that can be expressed as a radial part multiplied by a spherical harmonic:

$$
F_{\ell}^{m}(\boldsymbol{r})=f_{\ell}(r) Y_{\ell}^{m}(\boldsymbol{r} / r) .
$$

The straightforward differentiations of such an irreducible tensor of rank $\ell$ with respect to the Cartesian components $x, y$, and $z$ of $\boldsymbol{r}$ would in general lead to extremely messy expressions. Because of the convenient orthonormality properties of the spherical harmonics it would be advantageous if the angular part of such a product could be expressed in terms of spherical harmonics. Of course, it should be possible to do the necessary algebra, but in particular for large angular momentum quantum numbers we would be confronted with nontrivial technical problems.

Thus, we arrive at the paradoxical statement that the differential operator $\mathcal{Y}_{\ell}^{m}(\nabla)$ is practically useful only if it is not necessary to do differentiations with respect to $x, y$, and $z$ via the defining explicit expression (2.1). Fortunately, these differentiations can be avoided since $\mathcal{Y}_{\ell}^{m}(\nabla)$ is just like the corresponding regular solid harmonic $\mathcal{Y}_{\ell}^{m}(\boldsymbol{r})$ an irreducible spherical tensor

Ernst Joachim Weniger: The Spherical Tensor Gradient Operator 
of rank $\ell$ (compare [12, p. 312]). Consequently, matrix elements involving $\mathcal{Y}_{\ell}^{m}(\nabla)$ and other irreducible spherical tensors can be handled via the powerful machinery of angular momentum coupling.

As discussed in more details later, the product $\mathcal{Y}_{\ell_{1}}^{m_{1}}(\nabla) F_{\ell_{2}}^{m_{2}}(\boldsymbol{r})$ can be expressed as a finite linear combination of Gaunt coefficients defined in (C.1), radial functions $\gamma_{\ell_{1} \ell_{2}}^{\ell}(r)$, and spherical harmonics [131, Eq. (4.7)]:

$$
\begin{aligned}
\mathcal{Y}_{\ell_{1}}^{m_{1}}(\nabla) & F_{\ell_{2}}^{m_{2}}(\boldsymbol{r}) \\
& =\sum_{\ell=\ell_{\min }}^{\ell_{\max }}{ }^{(2)}\left\langle\ell m_{1}+m_{2}\left|\ell_{1} m_{1}\right| \ell_{2} m_{2}\right\rangle \gamma_{\ell_{1} \ell_{2}}^{\ell}(r) Y_{\ell}^{m_{1}+m_{2}}(\boldsymbol{r} / r) .
\end{aligned}
$$

The summation limits $\ell_{\min }$ and $\ell_{\max }$ are given by (C.5).

It is possible to derive alternatives to (2.3) which also take into account the tensorial nature of both $\mathcal{Y}_{\ell_{1}}^{m_{1}}(\nabla)$ and $F_{\ell_{2}}^{m_{2}}(\boldsymbol{r})$. For example, Bayman 6] derived an equivalent expression involving Clebsch-Gordan coefficients instead of Gaunt integrals as in (2.3). However, it was argued in 131, Section III] that $\nabla$ behaves like $\boldsymbol{r}$ under reflections. Accordingly, parity is conserved, and the replacement of Clebsch-Gordan coefficients by Gaunt integrals according to (C.2) leads to a considerable formal simplification.

As discussed in more details in Section 4 the functions $\gamma_{\ell_{1} \ell_{2}}^{\ell}(r)$ in (2.3) can be obtained by differentiating the radial part $f_{\ell_{2}}(r)$ of the spherical tensor $F_{\ell_{2}}^{m_{2}}(\boldsymbol{r})$ with respect to $r$ (compare the discussion in 131, Sections III and IV]). Thus, the systematic exploitation of the tensorial nature of $\mathcal{Y}_{\ell_{1}}^{m_{1}}(\nabla)$ makes it possible to replace potentially troublesome differentiations with respect to the three Cartesian components $x, y$, and $z$ by comparatively benign differentiations with respect to the radial variable $r$. This is a very important technical aspect since it greatly facilitates the application of the spherical tensor gradient operator to spherical tensors of the type of (2.2).

Other expressions for the product $\mathcal{Y}_{\ell_{1}}^{m_{1}}(\nabla) F_{\ell_{2}}^{m_{2}}(\boldsymbol{r})$ can be found in articles by Santos [95, Stuart [112, Niukkanen [70, and Rashid [83].

\section{Hobson's Theorem on Differentiation}

The first article on the spherical tensor gradient operator $\mathcal{Y}_{\ell}^{m}(\nabla)$, which I am aware of, is due to Hobson [42, p. 67] who derived in 1892 a very consequential theorem on the differentiation of functions $f: \mathbb{R}^{n} \rightarrow \mathbb{C}$. This theorem is also discussed in Hobson's book [43 pp. 124 - 129] that was first published in 1931.

Ernst Joachim Weniger: The Spherical Tensor Gradient Operator 
In contemporary articles dealing with the spherical tensor gradient operator, Hobson's theorem - Eq. (3.1) below - is in most cases completely ignored, but in the article by Bott, Methfessel, Krabs, and Schmidt 13. Section IV], the simplified form (3.2) of Hobson's theorem was even rederived in a less general form. This is an undeserved neglect. Firstly, many properties of the spherical tensor gradient operator $\mathcal{Y}_{\ell}^{m}(\nabla)$ can be deduced and understood easily via Hobson's theorem. Secondly, it should be possible to generalize the approach described in this article, which is based on the threedimensional regular solid harmonics $\mathcal{Y}_{\ell}^{m}(\boldsymbol{r})$, to differential operators based on $n$-dimensional hyperspherical harmonics. A problem of obvious physical relevance would be the construction of differential operators associated to the four-dimensional hyperspherical harmonics, which are for instance discussed in books by Avery [3, 4] and Judd [55, Sections 2 and 3 and Appendix 2 ] or also in [118, Section VI].

In three-dimensional form - which is all we need here - Hobson's theorem can be formulated as follows (compare also [129, Eq. (4.13)]):

Let $f_{n}(x, y, z)$ be a homogeneous polynomial of degree $n \in \mathbb{N}$ in the variables $x, y, z$, and let $F$ be any (differentiable) function that depends only on $r^{2}=x^{2}+y^{2}+z^{2}$. Then, the application of the differential operator $f_{n}(\partial / \partial x, \partial / \partial y, \partial / \partial z)$ to $F$ can be expressed in closed form according to

$$
\begin{aligned}
f_{n}\left(\frac{\partial}{\partial x}, \frac{\partial}{\partial y}, \frac{\partial}{\partial z}\right) F\left(r^{2}\right) \\
\quad=\sum_{\nu=0}^{n} \frac{2^{n-2 \nu}}{\nu !}\left[\left(\frac{\mathrm{d}}{\mathrm{d} r^{2}}\right)^{n-\nu} F\left(r^{2}\right)\right] \nabla^{2 \nu} f_{n}(x, y, z) .
\end{aligned}
$$

Let us now assume that $f_{n}$ is not only a homogeneous polynomial of degree $n$, but also a solution of the three-dimensional homogeneous Laplace equation (B.8). Then, (3.1) simplifies considerably because in the sum on the righthand side only the power $\nabla^{2 \nu}$ with $\nu=0$ produces a nonzero result:

$$
f_{n}\left(\frac{\partial}{\partial x}, \frac{\partial}{\partial y}, \frac{\partial}{\partial z}\right) F\left(r^{2}\right)=2^{n}\left[\left(\frac{\mathrm{d}}{\mathrm{d} r^{2}}\right)^{n} F\left(r^{2}\right)\right] f_{n}(x, y, z) .
$$

As discussed in Appendix $\mathrm{B}$, the regular solid harmonic $\mathcal{Y}_{\ell}^{m}(\boldsymbol{r})$ is a polynomial solution of the homogeneous Laplace equation (B.8) of degree $\ell$. Accordingly, Hobson's theorem applies in its simplified form (3.2), and we obtain (compare [129, Eq. (3.11)]):

$$
\mathcal{Y}_{\ell}^{m}(\nabla) F\left(r^{2}\right)=2^{\ell}\left[\left(\frac{\mathrm{d}}{\mathrm{d} r^{2}}\right)^{\ell} F\left(r^{2}\right)\right] \mathcal{Y}_{\ell}^{m}(\boldsymbol{r}) .
$$

Ernst Joachim Weniger: The Spherical Tensor Gradient Operator 
If we now set $F\left(r^{2}\right)=\varphi(r)$ and use $\mathrm{d} / \mathrm{d} r^{2}=1 /(2 r) \mathrm{d} / \mathrm{d} r$, we obtain (compare [129, Eq. (3.12)]):

$$
\mathcal{Y}_{\ell}^{m}(\nabla) \varphi(r)=\left[\left(\frac{1}{r} \frac{\mathrm{d}}{\mathrm{d} r}\right)^{\ell} \varphi(r)\right] \mathcal{Y}_{\ell}^{m}(\boldsymbol{r}) .
$$

It follows from either (3.3) or (3.4) that $\mathcal{Y}_{\ell}^{m}(\nabla)$ can be viewed as a generating differential operator which transforms a scalar function - an irreducible spherical tensor of rank 0 - to an irreducible spherical tensor of rank $\ell$.

Next, let us apply the spherical tensor gradient operator $\mathcal{Y}_{\ell_{1}}^{m_{1}}(\nabla)$ to irreducible spherical tensors $F_{\ell_{2}}^{m_{2}}$ of the type of (2.2) with nonzero rank $\ell_{2}$. To simplify things, let us for the moment assume that such a spherical tensor $F_{\ell_{2}}^{m_{2}}(\boldsymbol{r})$ satisfies a relation of the kind of (3.4), i.e., it can be generated by applying $\mathcal{Y}_{\ell_{2}}^{m_{2}}(\nabla)$ to a suitable scalar function $\Phi_{\ell_{2}}(r)$ :

$$
F_{\ell_{2}}^{m_{2}}(\boldsymbol{r})=\mathcal{Y}_{\ell_{2}}^{m_{2}}(\nabla) \Phi_{\ell_{2}}(r)
$$

In view of $\mathcal{Y}_{\ell_{1}}^{m_{1}}(\nabla) F_{\ell_{2}}^{m_{2}}(\boldsymbol{r})=\mathcal{Y}_{\ell_{1}}^{m_{1}}(\nabla) \mathcal{Y}_{\ell_{2}}^{m_{2}}(\nabla) \Phi_{\ell_{2}}(r)$, we need an explicit expression of manageable complexity for the product $\mathcal{Y}_{\ell_{1}}^{m_{1}}(\nabla) \mathcal{Y}_{\ell_{2}}^{m_{2}}(\nabla)$. This can be accomplished easily. If we multiply the linearization formula (C.4) of the surface harmonics by $r^{\ell_{1}+\ell_{2}}$, we obtain the linearization formula of the regular solid harmonics:

$$
\begin{aligned}
\mathcal{Y}_{\ell_{1}}^{m_{1}}(\boldsymbol{r}) & \mathcal{Y}_{\ell_{2}}^{m_{2}}(\boldsymbol{r}) \\
& =\sum_{\ell=\ell_{\min }}^{\ell_{\max }}{ }^{(2)}\left\langle\ell m_{1}+m_{2}\left|\ell_{1} m_{1}\right| \ell_{2} m_{2}\right\rangle r^{2 \Delta \ell} \mathcal{Y}_{\ell}^{m_{1}+m_{2}}(\boldsymbol{r}) .
\end{aligned}
$$

It follows at once from the summation limits (C.5) that the abbreviation $\Delta \ell$ defined by (C.6) is either a positive integer or zero. Accordingly, (3.6) can also be interpreted as a linearization formula for certain polynomials in the Cartesian components of an essentially arbitrary three-dimensional vector $\boldsymbol{r}$. Thus, (3.6) also holds for $\boldsymbol{r}=\nabla$, and we obtain (see for example [0, Eq. (15)], [5. Eq. (65)], or [129, Eq. (4.24)]):

$$
\begin{aligned}
\mathcal{Y}_{\ell_{1}}^{m_{1}}(\nabla) & \mathcal{Y}_{\ell_{2}}^{m_{2}}(\nabla) \\
& =\sum_{\ell=\ell_{\min }}^{\ell_{\max }}{ }^{(2)}\left\langle\ell m_{1}+m_{2}\left|\ell_{1} m_{1}\right| \ell_{2} m_{2}\right\rangle \nabla^{2 \Delta \ell} \mathcal{Y}_{\ell}^{m_{1}+m_{2}}(\nabla) .
\end{aligned}
$$

Ernst Joachim Weniger: The Spherical Tensor Gradient Operator 
If we now combine (3.5) and (3.7), we obtain an expression which is obviously of the form of (2.3) (see for example [133, Eq. (3.9)]):

$$
\begin{aligned}
\mathcal{Y}_{\ell_{1}}^{m_{1}}(\nabla) F_{\ell_{2}}^{m_{2}}(\boldsymbol{r})= & \sum_{\ell=\ell_{\min }}^{\ell_{\max }}(2)\left\langle\ell m_{1}+m_{2}\left|\ell_{1} m_{1}\right| \ell_{2} m_{2}\right\rangle \\
& \times \nabla^{2 \Delta \ell}\left[\left(\frac{1}{r} \frac{\mathrm{d}}{\mathrm{d} r}\right)^{\ell} \Phi_{\ell_{2}}(r)\right] \mathcal{Y}_{\ell}^{m_{1}+m_{2}}(\boldsymbol{r}) .
\end{aligned}
$$

There are some radially symmetric functions of considerable physical relevance which produce results of remarkable simplicity if $\mathcal{Y}_{\ell}^{m}(\nabla)$ is applied to them via (3.4). The classic example is the Coulomb potential $1 / r$. Hobson [42] showed that the irregular solid harmonic $\mathcal{Z}_{\ell}^{m}(\boldsymbol{r})$ defined in (B.7) is generated by applying $\mathcal{Y}_{\ell}^{m}(\nabla)$ to $1 / r$ (further details can be found in Hobson's book [43, pp. 124 - 129]). In modern notation, Hobson's result can be expressed as follows (see for example [129, Eq. (4.16)]):

$$
\mathcal{Z}_{\ell}^{m}(\boldsymbol{r})=\frac{(-1)^{\ell}}{(2 \ell-1) ! !} \mathcal{Y}_{\ell}^{m}(\nabla) \frac{1}{r}
$$

It is possible to derive this relationships differently (see for example 12, Chapter 6.18]), but its derivation via (3.4) is in my opinion much more straightforward and transparent.

It is also quite easy to obtain an explicit expression for the product $\mathcal{Y}_{\ell_{1}}^{m_{1}}(\nabla) \mathcal{Z}_{\ell_{2}}^{m_{2}}(\boldsymbol{r})$. With the help of (3.8) and (3.9), we immediately obtain the following compact expression involving Gaunt coefficients, Pochhammer symbols, and irregular solid harmonics [133, Eq. (4.7)]:

$$
\begin{aligned}
\mathcal{Y}_{\ell_{1}}^{m_{1}}(\nabla) \mathcal{Z}_{\ell_{2}}^{m_{2}}(\boldsymbol{r})= & \left\langle\ell_{1}+\ell_{2} m_{1}+m_{2}\left|\ell_{1} m_{1}\right| \ell_{2} m_{2}\right\rangle \\
& \times(-2)^{\ell_{1}} \frac{(1 / 2)_{\ell_{1}+\ell_{2}}}{(1 / 2)_{\ell_{2}}} \mathcal{Z}_{\ell_{1}+\ell_{2}}^{m_{1}+m_{2}}(\mathbf{r}) .
\end{aligned}
$$

Alternative derivations of this result are discussed in [120, Section 7].

In the vast majority of all LCAO-MO electronic structure calculations, Gaussian functions are used as basis functions. Traditionally, Cartesian Gaussian functions $x^{i} y^{j} z^{k} \exp \left(-\alpha r^{2}\right)$ with $i, j, k \in \mathbb{N}_{0}$ and $\alpha \in \mathbb{R}_{+}$have dominated, which indicates that the spherical tensor gradient operator should be irrelevant for calculations with Gaussian basis functions. However, in recent years research on molecular integrals of Gaussians has increasingly

Ernst Joachim Weniger: The Spherical Tensor Gradient Operator 
emphasized Gaussian functions whose angular parts are spherical harmonics. For these functions, $\mathcal{Y}_{\ell}^{m}(\nabla)$ is again an extremely useful mathematical tool (see for example the articles by Arakane and Matsuoka 1, Chow Chiu and Moharerrzadeh [16, 17, 18, Dunlap [22, 23, 24, 25, 26], Fieck 27. 28, Fortunelli and Carrravetta 32, Fortunelli and Salvetti 33, Fujimura and Matsuoka 34, Hu, Staufer, Birkenheuer, Igoshine, and Rösch [47], Ishida [48, 49, 50, 51, 52], Kuang and Lin [58, 59], Maretis [62, Matsuoka 63, 64, 65, 66, 67, and Saunders [96]).

The usefulness of $\mathcal{Y}_{\ell}^{m}(\nabla)$ in connection with Gaussian functions follows at once from the fact that $\exp \left(-\alpha r^{2}\right)$ is an eigenfunction of the differential operator $(1 / r) \mathrm{d} / \mathrm{d} r$ with eigenvalue $-2 \alpha$. Thus, the application of $\mathcal{Y}_{\ell}^{m}(\nabla)$ to $\exp \left(-\alpha r^{2}\right)$ via (3.4) yields according to Fieck [28] the following remarkably compact irreducible spherical tensor of rank $\ell$ :

$$
\mathcal{Y}_{\ell}^{m}(\nabla) \exp \left(-\alpha r^{2}\right)=(-2 \alpha)^{\ell} \exp \left(-\alpha r^{2}\right) \mathcal{Y}_{\ell}^{m}(\boldsymbol{r}) .
$$

A detailed discussion of the usefulness of the differential operator $\mathcal{Y}_{\ell}^{m}(\nabla)$ in the context of Gaussian functions and their multicenter integrals would certainly be of considerable interest, but unfortunately it would clearly be beyond the scope of this article. In addition, I cannot claim that molecular integrals of Gaussian functions are my field of expertise. Therefore, the interested reader is referred to the articles listed above.

Another class of functions, which have close ties to the differential operator $\mathcal{Y}_{\ell}^{m}(\nabla)$, are the s-called $B$ functions which will be discussed in a detailed way in Section 5 .

\section{Fourier Transformation}

As discussed in Section 3. Hobson's theorem implies that a spherical tensor of rank $\ell$ can be generated by applying $\mathcal{Y}_{\ell}^{m}(\nabla)$ to a spherically symmetric function $\varphi(r)$ according to (3.4). If an irreducible spherical tensor $F_{\ell_{2}}^{m_{2}}$ of the type of (2.2) also satisfies (3.5), then the simple explicit expression (3.8) for the product $\mathcal{Y}_{\ell_{1}}^{m_{1}}(\nabla) F_{\ell_{2}}^{m_{2}}(\boldsymbol{r})$ can be derived via (3.4).

In principle, (3.8) should suffice for our purposes, since the scalar function $\Phi_{\ell_{2}}(r)$ in (3.5) can according to (3.4) be obtained from the scalar function $f_{\ell_{2}}(r)$ in (2.2) by repeated integration with respect to $r$. However, repeated integrations are at least potentially a source of trouble, and it is thus desirable to express the product $\mathcal{Y}_{\ell_{1}}^{m_{1}}(\nabla) F_{\ell_{2}}^{m_{2}}(\boldsymbol{r})$ according to (2.3) in terms of

Ernst Joachim Weniger: The Spherical Tensor Gradient Operator 
radial functions $\gamma_{\ell_{1} \ell_{2}}^{\ell}(r)$ that can be obtained by differentiating the radial function $f_{\ell_{2}}(r)$ in (2.2). That this is indeed possible can be shown most easily with the help of Fourier transformation, which will be used in its symmetrical form. Thus, a function $f: \mathbb{R}^{3} \rightarrow \mathbb{C}$ and its Fourier transform $\bar{f}$ are connected by the integrals (A.2) and A.3.

The practical usefulness of Fourier transformation is obvious, and it would clearly be beyond the scope of this article to mention all successful scientific applications. Let me just mention that Fourier transformation is - as first shown by Prosser and Blanchard [82 and by Geller [37] - one of the principal methods of handling molecular multicenter integrals.

For example, the convolution of two functions $f, g: \mathbb{R}^{3} \rightarrow \mathbb{C}$ can be expressed as an inverse Fourier integral (see for example [131, Eq. (5.11)]):

$$
\int f\left(\boldsymbol{r}-\boldsymbol{r}^{\prime}\right) g\left(\boldsymbol{r}^{\prime}\right) \mathrm{d}^{3} \boldsymbol{r}^{\prime}=\int \mathrm{e}^{\mathrm{i} \boldsymbol{r} \cdot \boldsymbol{p}} \bar{f}(\boldsymbol{p}) \bar{g}(\boldsymbol{p}) \mathrm{d}^{3} \boldsymbol{p}
$$

We immediately obtain this result if we replace in (4.1) $f$ and $g$ by their inverse Fourier integrals according to A.3 and use the following integral representation of the three-dimensional delta function (see for example 55 . Eq. (3.16)]):

$$
\delta(\boldsymbol{r})=(2 \pi)^{-3} \int \mathrm{e}^{\mathrm{i} \boldsymbol{r} \cdot \boldsymbol{p}} \mathrm{d}^{3} \boldsymbol{p} .
$$

Six-dimensional integrals describing the Coulomb interaction of two charge densities $\left[f\left(\boldsymbol{r}_{1}\right)\right]^{*}$ and $g\left(\boldsymbol{r}_{2}\right)$ can also be expressed as three-dimensional inverse Fourier integrals (see for example [126, Eq. (2.22)]):

$$
\begin{gathered}
\iint\left[f\left(\boldsymbol{r}_{1}\right)\right]^{*} \frac{1}{\left|\boldsymbol{r}_{1}-\boldsymbol{r}_{2}-\boldsymbol{R}\right|} g\left(\boldsymbol{r}_{2}\right) \mathrm{d}^{3} \boldsymbol{r}_{1} \mathrm{~d}^{3} \boldsymbol{r}_{2} \\
=4 \pi \int \frac{\mathrm{e}^{-\mathrm{i} \boldsymbol{R} \cdot \boldsymbol{p}}}{p^{2}}[\bar{f}(\boldsymbol{p})]^{*} \bar{g}(\boldsymbol{p}) \mathrm{d}^{3} \boldsymbol{p}
\end{gathered}
$$

For a proof of (4.3), we also need the Fourier transform (6.4) of the Coulomb potential, which - as discussed in more details in Section 6 - holds only in the sense of distributions.

In connection with multicenter integrals in general and with the spherical tensor gradient operator in special, Fourier transformation suffers from a serious limitation which must not be ignored. Classically, Fourier transformation is defined only for functions that are absolutely integrable, i.e.,

Ernst Joachim Weniger: The Spherical Tensor Gradient Operator 
which belong to the function space $L^{1}\left(\mathbb{R}^{3}\right)$, but by means of a suitable limiting procedure it can be extended uniquely to give a unitary map from the Hilbert space $L^{2}\left(\mathbb{R}^{3}\right)$ of square integrable functions onto itself 84 , Theorem IX.6 on p. 10]. Unfortunately, not all functions of interest are square integrable and thus possess Fourier transforms that are meaningful in the sense of classical analysis. As discussed in more details in Section 6, the Coulomb potential and the irregular solid harmonic, which are connected via (3.9), possess Fourier transforms only in the sense of generalized functions or distributions. For the sake of conceptual simplicity, let us tacitly assume that all Fourier integrals in this Section are meaningful in the sense of classical analysis.

Fourier transformation greatly simplifies the treatment of the spherical tensor gradient operator because of the obvious relationship

$$
\mathcal{Y}_{\ell}^{m}(\nabla) \mathrm{e}^{\mathrm{i} \boldsymbol{r} \cdot \boldsymbol{p}}=\mathrm{i}^{\ell} \mathcal{Y}_{\ell}^{m}(\boldsymbol{p}) \mathrm{e}^{\mathrm{i} \boldsymbol{r} \cdot \boldsymbol{p}} .
$$

Thus, in momentum space $\mathcal{Y}_{\ell}^{m}(\nabla)$ is a simple multiplicative operator with known coupling properties. Moreover, (4.4) makes it plausible that $\mathcal{Y}_{\ell}^{m}(\nabla)$ is indeed an irreducible spherical tensor of rank $\ell$ (compare [12, p. 312]).

If we use in the Fourier integral (A.2) both (2.2) as well as the Rayleigh expansion of a plane wave in terms of spherical Bessel functions and spherical harmonics (compare for instance [12, p. 442]),

$$
\mathrm{e}^{ \pm \mathrm{i} \boldsymbol{p} \cdot \boldsymbol{r}}=4 \pi \sum_{\ell=0}^{\infty}( \pm 1)^{\ell} j_{\ell}(p r) \sum_{m=-\ell}^{\ell}\left[Y_{\ell}^{m}(\boldsymbol{p} / p)\right]^{*} Y_{\ell}^{m}(\boldsymbol{r} / r),
$$

we see that the Fourier transform $\bar{F}_{\ell_{2}}^{m_{2}}(\boldsymbol{p})$ of the irreducible spherical tensor $F_{\ell_{2}}^{m_{2}}(\boldsymbol{r})$ is also a spherical tensor of rank $\ell_{2}$ in momentum space since it can be expressed as a spherical harmonic multiplied by a radial integral [131. Eqs. (4.3) and (4.4)]:

$$
\begin{aligned}
\bar{F}_{\ell_{2}}^{m_{2}}(\boldsymbol{p}) & =\bar{f}_{\ell_{2}}(p) Y_{\ell_{2}}^{m_{2}}(\boldsymbol{p} / p), \\
\bar{f}_{\ell_{2}}(p) & =(-\mathrm{i})^{\ell_{2}} p^{-1 / 2} \int_{0}^{\infty} r^{3 / 2} J_{\ell_{2}+1 / 2}(p r) f_{\ell_{2}}(r) \mathrm{d} r .
\end{aligned}
$$

In the same way, we obtain the following Hankel-type integral representation for the radial part $f_{\ell_{2}}(r)$ of $F_{\ell_{2}}^{m_{2}}(\boldsymbol{r})$ [131, Eq. (4.5)]:

$$
f_{\ell_{2}}(r)=\mathrm{i}^{\ell_{2}} r^{-1 / 2} \int_{0}^{\infty} p^{3 / 2} J_{\ell_{2}+1 / 2}(r p) \bar{f}_{\ell_{2}}(p) \mathrm{d} p .
$$

Ernst Joachim Weniger: The Spherical Tensor Gradient Operator 
Let us now consider the product $\mathcal{Y}_{\ell_{1}}^{m_{1}}(\nabla) F_{\ell_{2}}^{m_{2}}(\boldsymbol{r})$. With the help of (4.4) and (A.3), we obtain the following Fourier integral representation:

$$
\mathcal{Y}_{\ell_{1}}^{m_{1}}(\nabla) F_{\ell_{2}}^{m_{2}}(\boldsymbol{r})=\mathrm{i}^{\ell_{1}}(2 \pi)^{-3 / 2} \int \mathrm{e}^{\mathrm{i} \boldsymbol{r} \cdot \boldsymbol{p}} \mathcal{Y}_{\ell_{1}}^{m_{1}}(\boldsymbol{p}) \bar{F}_{\ell_{2}}^{m_{2}}(\boldsymbol{p}) \mathrm{d}^{3} \boldsymbol{p} .
$$

Next, we use the Rayleigh expansion (4.5) and replace the spherical harmonics by Gaunt coefficients according to (C.4). This yields [131, Eq. (4.7)]:

$$
\begin{aligned}
\mathcal{Y}_{\ell_{1}}^{m_{1}}(\nabla) & F_{\ell_{2}}^{m_{2}}(\boldsymbol{r}) \\
= & \sum_{\ell=\ell_{\min }}^{\ell_{\max }}{ }^{(2)}\left\langle\ell m_{1}+m_{2}\left|\ell_{1} m_{1}\right| \ell_{2} m_{2}\right\rangle \mathrm{i}^{\ell+\ell_{1}} Y_{\ell}^{m_{1}+m_{2}}(\boldsymbol{r} / r) \\
& \times r^{-1 / 2} \int_{0}^{\infty} p^{\ell_{1}+3 / 2} J_{\ell+1 / 2}(r p) \bar{f}_{\ell_{2}}(p) \mathrm{d} p .
\end{aligned}
$$

Comparison of (2.3) and (4.9) yields the following integral representation for the radial function $\gamma_{\ell_{1} \ell_{2}}^{\ell}(r)$ [131, Eq. (4.8)]:

$$
\gamma_{\ell_{1} \ell_{2}}^{\ell}(r)=\mathrm{i}^{\ell+\ell_{1}} r^{-1 / 2} \int_{0}^{\infty} p^{\ell_{1}+3 / 2} J_{\ell+1 / 2}(r p) \bar{f}_{\ell_{2}}(p) \mathrm{d} p .
$$

Now, all we need is a differential operator in $r$ that generates the integral in (4.10) from the integral in (4.7). With the help of known properties of the Bessel function $J_{\nu}(z)$ or by direct differentiation techniques (see 131, Section III]), this can be accomplished relatively easily [131, Eqs. (3.29), (4.15) - (4.18), and (4.24)]:

$$
\begin{aligned}
\gamma_{\ell_{1} \ell_{2}}^{\ell}(r)= & \sum_{q=0}^{\Delta \ell} \frac{(-\Delta \ell)_{q}(-\sigma(\ell)-1 / 2)_{q}}{q !} 2^{q} r^{\ell_{1}+\ell_{2}-2 q} \\
& \times\left(\frac{1}{r} \frac{\mathrm{d}}{\mathrm{d} r}\right)^{\ell_{1}-q} \frac{f_{\ell_{2}}(r)}{r^{\ell_{2}}} \\
= & r^{-\ell-1}\left(\frac{1}{r} \frac{\mathrm{d}}{\mathrm{d} r}\right)^{\Delta \ell} r^{\ell_{1}+\ell_{2}+\ell+1}\left(\frac{1}{r} \frac{\mathrm{d}}{\mathrm{d} r}\right)^{\Delta \ell_{2}} \frac{f_{\ell_{2}}(r)}{r^{\ell_{2}}} \\
= & r^{\ell}\left(\frac{1}{r} \frac{\mathrm{d}}{\mathrm{d} r}\right)^{\Delta \ell_{2}} r^{\ell_{1}-\ell_{2}-\ell-1}\left(\frac{1}{r} \frac{\mathrm{d}}{\mathrm{d} r}\right)^{\Delta \ell} r^{\ell_{2}+1} f_{\ell_{2}}(r) \\
= & r^{-\ell-1}\left(\frac{1}{r} \frac{\mathrm{d}}{\mathrm{d} r}\right)^{\Delta \ell_{2}} r^{\ell_{1}-\ell_{2}+3 \ell+1}\left(\frac{1}{r} \frac{\mathrm{d}}{\mathrm{d} r}\right)^{\Delta \ell_{2}}
\end{aligned}
$$

Ernst Joachim Weniger: The Spherical Tensor Gradient Operator 


$$
\begin{aligned}
& \times r^{-2 \ell-1}\left(\frac{1}{r} \frac{\mathrm{d}}{\mathrm{d} r}\right)^{\ell_{2}-\ell} r^{\ell_{2}+1} f_{\ell_{2}}(r) \\
= & r^{\ell}\left(\frac{1}{r} \frac{\mathrm{d}}{\mathrm{d} r}\right)^{\Delta \ell} r^{\ell_{1}+\ell_{2}-3 \ell-1}\left(\frac{1}{r} \frac{\mathrm{d}}{\mathrm{d} r}\right)^{\Delta \ell} \\
& \times r^{2 \ell+1}\left(\frac{1}{r} \frac{\mathrm{d}}{\mathrm{d} r}\right)^{\ell-\ell_{2}} \frac{f_{\ell_{2}}(r)}{r^{\ell_{2}}} \\
= & \sum_{s=0}^{\Delta \ell_{2}} \frac{\left(-\Delta \ell_{2}\right)_{s}\left(\Delta \ell_{1}+1 / 2\right)_{s}}{s !} 2^{s} r^{\ell_{1}-\ell_{2}-2 s-1} \\
& \times\left(\frac{1}{r} \frac{\mathrm{d}}{\mathrm{d} r}\right)^{\ell_{1}-s} r^{\ell_{2}+1} f_{\ell_{2}}(r) .
\end{aligned}
$$

The abbreviations $\Delta l, \Delta l_{1}, \Delta l_{2}$, and $\sigma(\ell)$ are defined in (C.6) - C.9. Obviously, the representations (4.14) and (4.15) make sense only if either $\ell_{2} \geq \ell$ or $\ell \geq \ell_{2}$ hold.

The expressions (4.11) - (4.16) for $\gamma_{\ell_{1} \ell_{2}}^{\ell}(r)$ all look quite different. Nevertheless, their equivalence can be shown explicitly with the help of summation theorems for generalized hypergeometric series with unit argument (see the discussion in connection with [131, Eqs. (4.19) - (4.24)]).

Alternative expressions for $\gamma_{\ell_{1} \ell_{2}}^{\ell}(r)$ as well as for more general radial functions, that occur in the case of the product $\nabla^{2 n} \mathcal{Y}_{\ell_{1}}^{m_{2}}(\nabla) F_{\ell_{2}}^{m_{2}}(\boldsymbol{r})$ with $n \in \mathbb{N}_{0}$, were considered by Santos [95], Bayman [6], Stuart [112, Niukkanen [70], Weniger and Steinborn [131, and Rashid [83.

The expressions (4.11) - 4.16) for $\gamma_{\ell_{1} \ell_{2}}^{\ell}(r)$ all have a manageable complexity and are well suited for practical applications. Nevertheless, it is more convenient to compute the product $\mathcal{Y}_{\ell_{1}}^{m_{2}}(\nabla) F_{\ell_{2}}^{m_{2}}(\boldsymbol{r})$ or also the more general product $\nabla^{2 n} \mathcal{Y}_{\ell_{1}}^{m_{2}}(\nabla) F_{\ell_{2}}^{m_{2}}(\boldsymbol{r})$ with $n \in \mathbb{N}_{0}$ via (3.8) if the function $\Phi_{\ell_{2}}(r)$ defined in (3.5) is easily accessible and has a sufficiently simple form as in the case of the irregular solid harmonic and the Gaussian function according to (3.9) and (3.11), respectively, or in the case of $B$ functions according to (5.9).

With the help of Fourier transformation, it is easy to obtain the explicit expression for the product $\mathcal{Y}_{\ell}^{m}(\nabla) f(\boldsymbol{r}) g(\boldsymbol{r})$ which could be called the Leibniz theorem of the spherical tensor gradient operator and which was originally derived by Dunlap [22, Eq. (10)], albeit in a somewhat cryptic way. My derivation is inspired by Grotendorst and Steinborn [39, Appendix A] who

Ernst Joachim Weniger: The Spherical Tensor Gradient Operator 
had derived a more general expression involving spherical tensor gradient operators in connection with the Fourier transform of a two-center density.

For that purpose, let us express both $f$ and $g$ as inverse Fourier integrals according to (A.3) and use (4.4):

$$
\begin{aligned}
& \mathcal{Y}_{\ell}^{m}(\nabla) f(\boldsymbol{r}) g(\boldsymbol{r})=\frac{\mathcal{Y}_{\ell}^{m}(\nabla)}{(2 \pi)^{3}} \iint \mathrm{e}^{\mathrm{i} \boldsymbol{r} \cdot(\boldsymbol{p}+\boldsymbol{q})} \bar{f}(\boldsymbol{p}) \bar{g}(\boldsymbol{p}) \mathrm{d}^{3} \boldsymbol{p} \mathrm{d}^{3} \boldsymbol{q} \\
&=(2 \pi)^{-3} \iint \mathrm{e}^{\mathrm{i} \boldsymbol{r} \cdot(\boldsymbol{p}+\boldsymbol{q})} \mathcal{Y}_{\ell}^{m}(\mathrm{i}[\boldsymbol{p}+\boldsymbol{q}]) \bar{f}(\boldsymbol{p}) \bar{g}(\boldsymbol{p}) \mathrm{d}^{3} \boldsymbol{p} \mathrm{d}^{3} \boldsymbol{q} .
\end{aligned}
$$

The dependence of the regular spherical harmonic on the two momentum variables $\boldsymbol{p}$ and $\boldsymbol{q}$ can be decoupled with the help of the well known addition theorem of the regular solid harmonics (a particularly simple derivation of this addition theorem based on the the spherical tensor gradient operator can be found in [120, Section 6]):

$$
\begin{aligned}
\mathcal{Y}_{\ell}^{m}\left(\boldsymbol{r}+\boldsymbol{r}^{\prime}\right)= & \sum_{\lambda=0}^{\ell} \frac{2 \pi(1 / 2)_{\ell+1}}{(1 / 2)_{\lambda+1}(1 / 2)_{\ell-\lambda+1}} \\
& \times \sum_{\mu=-\lambda}^{\lambda}\langle\ell m|\lambda-\mu| \ell-\lambda m+\mu\rangle \mathcal{Y}_{\lambda}^{-\mu}(\boldsymbol{r}) \mathcal{Y}_{\ell-\lambda}^{m+\mu}\left(\boldsymbol{r}^{\prime}\right) .
\end{aligned}
$$

The Gaunt coefficients in this addition theorem can be expressed in closed form (compare for instance [120, Eq. (6.5)] which unfortunately contains a typographical error: The factor $\left(2 \ell_{2}-\ell_{1}+1\right)$ in the numerator of the square root on the right-hand side has to be replaced by $\left(2 \ell_{2}-2 \ell_{1}+1\right)$.) In this way, we obtain Steinborn's factor-free version of the addition theorem of the regular solid harmonics [110, Eq. (9)].

If we now insert (4.19) into (4.18) and use (4.4), we obtain:

$$
\begin{aligned}
\mathcal{Y}_{\ell}^{m}(\nabla) & f(\boldsymbol{r}) g(\boldsymbol{r}) \\
= & \sum_{\lambda=0}^{\ell} \frac{2 \pi(1 / 2)_{\ell+1}}{(1 / 2)_{\lambda+1}(1 / 2)_{\ell-\lambda+1}} \sum_{\mu=-\lambda}^{\lambda}\langle\ell m|\lambda-\mu| \ell-\lambda m+\mu\rangle \\
& \times(2 \pi)^{-3 / 2} \int \mathrm{e}^{\mathrm{i} \boldsymbol{r} \cdot \boldsymbol{p}} \mathcal{Y}_{\lambda}^{-\mu}(\mathrm{i} \boldsymbol{p}) \bar{f}(\boldsymbol{p}) \mathrm{d}^{3} \boldsymbol{p} \\
& \times(2 \pi)^{-3 / 2} \int \mathrm{e}^{\mathrm{i} \boldsymbol{r} \cdot \boldsymbol{q}} \mathcal{Y}_{\ell-\lambda}^{m+\mu}(\mathrm{i} \boldsymbol{q}) \bar{g}(\boldsymbol{q}) \mathrm{d}^{3} \boldsymbol{q}
\end{aligned}
$$

Ernst Joachim Weniger: The Spherical Tensor Gradient Operator 


$$
\begin{aligned}
& =\sum_{\lambda=0}^{\ell} \frac{2 \pi(1 / 2)_{\ell+1}}{(1 / 2)_{\lambda+1}(1 / 2)_{\ell-\lambda+1}} \sum_{\mu=-\lambda}^{\lambda}\langle\ell m|\lambda-\mu| \ell-\lambda m+\mu\rangle \\
& \quad \times\left[(2 \pi)^{-3 / 2} \mathcal{Y}_{\lambda}^{-\mu}(\nabla) \int \mathrm{e}^{\mathrm{i} \boldsymbol{r} \cdot \boldsymbol{p}} \bar{f}(\boldsymbol{p}) \mathrm{d}^{3} \boldsymbol{p}\right] \\
& \quad \times\left[(2 \pi)^{-3 / 2} \mathcal{Y}_{\ell-\lambda}^{m+\mu}(\nabla) \int \mathrm{e}^{\mathrm{i} \boldsymbol{r} \cdot \boldsymbol{q}} \bar{g}(\boldsymbol{q}) \mathrm{d}^{3} \boldsymbol{q}\right] \\
& =\sum_{\lambda=0}^{\ell} \frac{2 \pi(1 / 2)_{\ell+1}}{(1 / 2)_{\lambda+1}(1 / 2)_{\ell-\lambda+1}} \sum_{\mu=-\lambda}^{\lambda}\langle\ell m|\lambda-\mu| \ell-\lambda m+\mu\rangle \\
& \quad \times\left[\mathcal{Y}_{\lambda}^{-\mu}(\nabla) f(\boldsymbol{r})\right]\left[\mathcal{Y}_{\ell-\lambda}^{m+\mu}(\nabla) g(\boldsymbol{r})\right] .
\end{aligned}
$$

Thus, the Leibniz theorem (4.22) of the spherical tensor gradient operator is nothing but the addition theorem (4.19) of the regular solid harmonic in momentum space.

\section{$5 \quad$ Reduced Bessel Functions}

In Section 3 it was shown that the application of the spherical tensor gradient operator $\mathcal{Y}_{\ell}^{m}(\nabla)$ to the Coulomb potential or to a scalar Gaussian yields remarkably compact results according to (3.9) or (3.11), respectively.

Other functions, to which the spherical tensor gradient operator can be applied with remarkable ease, are the so-called reduced Bessel functions, whose use in quantum chemistry had been proposed by Shavitt 101, Eq. (55) on p. 15], and their anisotropic generalizations, the so-called $B$ functions, which had been introduced by Filter and Steinborn [30, Eq. (2.14)]. Detailed discussions of the mathematical properties of reduced Bessel functions and $B$ functions can be found in my $\mathrm{PhD}$ thesis 117 and in the $\mathrm{PhD}$ thesis of Homeier 44.

If $K_{\nu}(z)$ is a modified Bessel function of the second kind [61, p. 66], the reduced Bessel function is defined by [103, Eqs. (3.1) and (3.2)]

$$
\hat{k}_{\nu}(z)=(2 / \pi)^{1 / 2} z^{\nu} K_{\nu}(z) .
$$

If the order $\nu$ of a reduced Bessel function is half-integral, $\nu=n+1 / 2$ with $n \in \mathbb{N}_{0}$, the reduced Bessel function can be written as an exponential multiplied by a terminating confluent hypergeometric series ${ }_{1} F_{1}$ [130, Eq. $(3.7)]$ :

$$
\hat{k}_{n+1 / 2}(z)=2^{n}(1 / 2)_{n} \mathrm{e}^{-z}{ }_{1} F_{1}(-n ;-2 n ; 2 z) .
$$

Ernst Joachim Weniger: The Spherical Tensor Gradient Operator 
The polynomial part in (5.2) was also treated independently in the mathematical literature, where the notation $\Theta_{n}(z)=\mathrm{e}^{z} \hat{k}_{n+1 / 2}(z)$ is used. Together with some other, closely related polynomials, the $\Theta_{n}(z)$ are called Bessel polynomials [38. According to Grosswald 38, they are applied in such diverse fields as number theory, statistics, and the analysis of complex electrical networks.

The Bessel polynomials $\Theta_{n}(z)$ occur also in a completely different mathematical context: In the book by Baker and Graves-Morris [5, p. 8] on Padé approximants, it is remarked that Padé had shown in his seminal thesis 81 that the Padé approximant $[n / m]$ to the exponential function $\exp (z)$ can be expressed as the ratio of two terminating confluent hypergeometric series 5 , Eq. (2.12)]:

$$
[n / m]=\frac{{ }_{1} F_{1}(-n ;-n-m ; z)}{{ }_{1} F_{1}(-n ;-n-m ;-z)}, \quad n, m \in \mathbb{N}_{0} .
$$

Accordingly, the diagonal Padé approximant with $n=m$ to the exponential function can be expressed as the ratio of two Bessel polynomials:

$$
[n / n]=\frac{\theta_{n}(z / 2)}{\theta_{n}(-z / 2)}, \quad n \in \mathbb{B}_{0} .
$$

As an anisotropic generalization of the reduced Bessel function with halfintegral order, the so-called $B$ function was introduced by Filter and Steinborn [30, Eq. (2.14)],

$$
B_{n, \ell}^{m}(\alpha, \boldsymbol{r})=\left[2^{n+\ell}(n+\ell) !\right]^{-1} \hat{k}_{n-1 / 2}(\alpha r) \mathcal{Y}_{\ell}^{m}(\alpha \boldsymbol{r}), \quad \alpha>0, n \in \mathbb{Z} .
$$

$B$ functions are a fairly large class of exponentially decaying functions. For $n \in \mathbb{N}$, they are suited to serve as trial functions in LCAO-MO calculations. Since, however, $B$ functions have a much more complicated mathematical structure than for example Slater-type functions, whose molecular multicenter integrals functions are notoriously difficult, it is by no means obvious that anything can be gained by using $B$ functions instead of Slatertype functions as basis functions. However, $B$ functions possess a Fourier transform of remarkable simplicity:

$$
\begin{aligned}
\bar{B}_{n, \ell}^{m}(\alpha, \boldsymbol{p}) & =(2 \pi)^{-3 / 2} \int \mathrm{e}^{-\mathrm{i} \boldsymbol{p} \cdot \boldsymbol{r}} B_{n, \ell}^{m}(\alpha, \boldsymbol{r}) \mathrm{d}^{3} \boldsymbol{r} \\
& =(2 / \pi)^{1 / 2} \frac{\alpha^{2 n+\ell-1}}{\left[\alpha^{2}+p^{2}\right]^{n+\ell+1}} \mathcal{Y}_{\ell}^{m}(-i \boldsymbol{p}) .
\end{aligned}
$$

Ernst Joachim Weniger: The Spherical Tensor Gradient Operator 
This is most likely the most consequential and certainly the most often cited result of my PhD thesis [117, Eq. (7.1-6) on p. 160]. Later, (5.6) was published in [129, Eq. (3.7)]. Independently and almost simultaneously, the Fourier transform (5.6) was also derived by Niukkanen [72].

The exceptionally simple Fourier transform (5.6) gives $B$ functions a unique position among exponentially decaying basis functions, and it also explains why other exponentially decaying functions like Slater-type functions, bound state hydrogen eigenfunctions or other functions sets based on generalized Laguerre polynomials can all be expressed in terms of finite linear combinations of $B$ functions (details and further references can found in [118, Section IV] or [122, Section 4]).

As discussed in Section 4. Fourier transformation is one of the principal approaches for the treatment of multicenter integrals [82, 37.

Thus, the simplicity of the Fourier transform (5.6) makes it plausible that multicenter integrals of $B$ functions can normally be evaluated more easily than the analogous integrals of other exponentially decaying functions as for instance Slater-type functions, For example, by inserting (5.6) into the inverse Fourier integral on the right-hand side of (4.1), we immediately obtain the following, extremely simple expression for the convolution integral of two $B$ functions with equal scaling parameters:

$$
\begin{aligned}
\int B_{n_{1}, \ell_{1}}^{m_{1}}\left(\alpha,\left[\boldsymbol{r}-\boldsymbol{r}^{\prime}\right]\right) B_{n_{2}, \ell_{2}}^{m_{2}}\left(\alpha, \boldsymbol{r}^{\prime}\right) \mathrm{d} \boldsymbol{r}^{\prime} \\
=\frac{4 \pi}{\alpha^{3}} \sum_{\ell=\ell_{\min }}^{\ell_{\max }}(2)\left\langle m_{1}+m_{2}\left|\ell_{1} m_{1}\right| \ell_{2} m_{2}\right\rangle \\
\quad \times \sum_{t=0}^{\Delta \ell}(-1)^{t}\left(\begin{array}{c}
\Delta \ell \\
t
\end{array}\right) B_{n_{1}+n_{2}+\ell_{1}+\ell_{2}-\ell-t+1, \ell}^{m_{1}+m_{2}}(\alpha, \boldsymbol{r}) .
\end{aligned}
$$

This expression was originally derived by Filter and Steinborn 29, Eq. (4.1)] with the help of an addition theorem. The summation limits $\ell_{\min }$ and $\ell_{\max }$ are given in (C.5), and $\Delta \ell$ is defined by (C.6).

In recent years, some significant progress has been achieved with respect to molecular multicenter integrals of $B$ functions (see for example the articles by Steinborn, Homeier, Fernández Rico, Ema, López, and Ramírez [105], and Steinborn, Homeier, Ema, López, and Ramírez [104]). Particularly promising seems to be the approach of Safouhi who - starting from the Fourier transform (5.6) - converts complicated multicenter integrals of $B$ or

Ernst Joachim Weniger: The Spherical Tensor Gradient Operator 
Slater-type functions to multi-dimensional integral representations involving nonphysical variables that have to be evaluated by numerical quadrature. At first sight, this does not look like a good idea because of the oscillatory nature of the multi-dimensional integral representations, which makes the straightforward application of conventional quadrature methods difficult. However, these computational problems can be overcome if the quadrature schemes are combined with suitable nonlinear extrapolation methods. Based on previous work of Sidi[102] and of Levin and Sidi [60] on extrapolation methods for numerical quadrature schemes, Safouhi succeeded in developing some extrapolation techniques specially suited for his needs. This permits a remarkably efficient and reliable evaluation of complicated molecular multicenter integrals via oscillatory (Fourier based) integral representations (see for example the recent articles by Berlu and Safouhi [8, 9, 10, Berlu, Safouhi and Hoggan 11], Safouhi [86, 87, 88, 89, 90, 91, Safouhi and Hoggan 92, 93, 94] and references therein). Safouhi's work can also be viewed as a convincing demonstration of the practical usefulness of extrapolation and convergence acceleration techniques in molecular electronic structure theory.

It follows at once from (5.6) that a $B$ function can be expressed as an inverse Fourier integral according to

$$
B_{n, \ell}^{m}(\alpha, \boldsymbol{r})=\frac{\alpha^{2 n+\ell-1}}{2 \pi^{2}} \int \mathrm{e}^{\mathrm{i} \boldsymbol{r} \cdot \boldsymbol{p}} \frac{\mathcal{Y}_{\ell}^{m}(-\mathrm{i} \boldsymbol{p})}{\left[\alpha^{2}+p^{2}\right]^{n+\ell+1}} \mathrm{~d}^{3} \boldsymbol{p} .
$$

Comparison of (4.4) and (5.8) shows that the application of the spherical tensor gradient operator to a scalar $B$ function yields a nonscalar $B$ function [117, Eq. (7.1-10) on p. 161]:

$$
B_{n, \ell}^{m}(\alpha, \boldsymbol{r})=(4 \pi)^{1 / 2}(-\alpha)^{-\ell} \mathcal{Y}_{\ell}^{m}(\nabla) B_{n+\ell, 0}^{0}(\alpha, \boldsymbol{r}) .
$$

This as well as several other related results can also be deduced from known properties of the modified Bessel function $K_{\nu}(z)$ via (3.4) (see for example [129, Eq. (4.12)]):

It is also quite easy to derive an explicit expression for the product $\mathcal{Y}_{\ell_{1}}^{m_{1}}(\nabla) B_{n_{2}, \ell_{2}}^{m_{2}}(\boldsymbol{r})$ via (3.8) since the application of higher powers of the Laplacian $\nabla^{2}$ to a $B$ function poses no problems. It follows at once from the integral representation (5.8) that the differential operator $1-\alpha^{-2} \nabla^{2}$ of the modified Helmholtz equation functions as a ladder operator [131, Eq. (5.6)]:

$$
\left[1-\alpha^{-2} \nabla^{2}\right] B_{n, \ell}^{m}(\alpha, \boldsymbol{r})=B_{n-1, \ell}^{m}(\alpha, \boldsymbol{r}) .
$$

Ernst Joachim Weniger: The Spherical Tensor Gradient Operator 
As discussed in more details in Section [6 this relationship holds also if the indices $n$ and $\ell$ satisfies $n+\ell<0$, i.e., for $B$ functions that are derivatives of the three-dimensional delta function according to (6.19).

Thus, the binomial expansion of $\alpha^{-2 \nu} \nabla^{2 \nu}$ in powers of $1-\alpha^{-2} \nabla^{2}$ in combination with (5.10) yields [131, Eq. (5.7)]:

$$
\alpha^{-2 \nu} \nabla^{2 \nu} B_{n, \ell}^{m}(\alpha, \boldsymbol{r})=\sum_{t=0}^{\nu}(-1)^{t}\left(\begin{array}{l}
\nu \\
t
\end{array}\right) B_{n-t, \ell}^{m}(\alpha, \boldsymbol{r}) .
$$

If we now combine (3.8) with (5.11), we immediately obtain the following compact linear combination of Gaunt coefficients, binomial coefficients, and $B$ functions [131, Eq. (6.25)]:

$$
\begin{aligned}
\mathcal{Y}_{\ell_{1}}^{m_{1}}(\nabla) B_{n_{2}, \ell_{2}}^{m_{2}}(\alpha, \boldsymbol{r})= & (-\alpha)^{\ell_{1}} \sum_{\ell=\ell_{\min }}^{\ell_{\max }}(2)\left\langle\ell m_{1}+m_{2}\left|\ell_{1} m_{1}\right| \ell_{2} m_{2}\right\rangle \\
& \times \sum_{t=0}^{\Delta \ell}(-1)^{t}\left(\begin{array}{c}
\Delta \ell \\
t
\end{array}\right) B_{n_{2}+\ell_{2}-\ell-t, \ell}^{m_{1}+m_{2}}(\alpha, \boldsymbol{r}) .
\end{aligned}
$$

It follows from the summation limits (C.5) that $\Delta \ell$ defined in (C.6) is either a positive integer or zero.

As already remarked above, all the commonly used exponentially decaying functions like Slater-type functions, bound state hydrogen eigenfunctions or other functions sets based on generalized Laguerre polynomials can all be expressed in terms of finite linear combinations of $B$ functions (see for example [118, Section IV] or [122, Section 4]). Thus, it follows from (5.12) that the product of a spherical tensor gradient operator and one of these exponentially decaying functions can be expressed as a finite linear combination of $B$ functions.

\section{Spherical Delta Functions}

Classically, the domain of the spherical tensor gradient operator consists of the differentiable functions $f: \mathbb{R}^{3} \rightarrow \mathbb{C}$, although we are in practice only interested in differentiable irreducible spherical tensors of the type of (2.2). However, as for example argued in Dirac's classic book [21, §15], the functions defined in the sense of classical analysis do not suffice in quantum theory. It is necessary to use also more general mathematical objects, the

Ernst Joachim Weniger: The Spherical Tensor Gradient Operator 
so-called generalized functions or distributions, whose mathematical theory was rigorously formulated by Schwartz (see 99] and references therein).

The best known nonclassical generalization of a function $f: \mathbb{R}^{3} \rightarrow \mathbb{C}$ is the three-dimensional Dirac delta function that can be defined as a generalized solution of the Poisson equation of a unit point charge [53, Eq. (1.31)]:

$$
\nabla^{2} \frac{1}{r}=-4 \pi \delta(\boldsymbol{r})
$$

This Poisson equation also expresses the well known fact that the Coulomb potential is the Green's function of the three-dimensional Laplace equation.

The prototype of a distribution, which is also an irreducible spherical tensor of rank $\ell$, is the so-called spherical delta function (see for example [85. Eq. (30)]):

$$
\delta_{\ell}^{m}(\boldsymbol{r})=\frac{(-1)^{\ell}}{(2 \ell-1) ! !} \mathcal{Y}_{\ell}^{m}(\nabla) \delta(\boldsymbol{r})
$$

The spherical delta function can also be obtained by applying the Laplacian to an irregular solid harmonics (see for example [85, Eq. (29)]):

$$
\nabla^{2} \mathcal{Z}_{\ell}^{m}(\boldsymbol{r})=-4 \pi \delta_{\ell}^{m}(\boldsymbol{r})
$$

This follows at once from (3.9), (6.1), (6.2), and the fact that $\nabla^{2}$ and $\mathcal{Y}_{\ell}^{m}(\nabla)$ commute. Comparison of (6.1) and (6.3) shows that the spherical delta function can be viewed as a generalized solution of the Poisson equation of a unit multipole charge.

The properties of generalized functions of the type of the spherical delta function can be understood most easily with the help of Fourier transformation. As already mentioned in Section 4. Fourier transformation is defined in the sense of classical analysis only for functions that are absolutely integrable, i.e., which belong to the function space $L^{1}\left(\mathbb{R}^{3}\right)$. By means of a suitable limiting procedure, Fourier transformation can be extended uniquely to give a unitary map from the Hilbert space $L^{2}\left(\mathbb{R}^{3}\right)$ of square integrable functions onto itself (see for example [84, Theorem IX.6 on p. 10]). A further extension of Fourier transformation to the space of tempered distributions is also possible (see for example [84, Theorem IX.2 on p. 5]).

The extensibility of Fourier transformation to nonclassical function spaces is very important for our purposes. For example, the Coulomb potential is neither absolutely integrable nor square integrable. Nevertheless, it is possible to define its Fourier transform in the sense of distributions [36, Eq. (2)

Ernst Joachim Weniger: The Spherical Tensor Gradient Operator 
on p. 194]:

$$
\frac{(2 / \pi)^{1 / 2}}{p^{2}}=(2 \pi)^{-3 / 2} \int \frac{\mathrm{e}^{-\mathrm{i} \boldsymbol{p} \cdot \boldsymbol{r}}}{r} \mathrm{~d}^{3} \boldsymbol{r} .
$$

This relationship makes it possible to convert multicenter integrals, which describe the Coulomb interaction of classical or nonclassical charge densities, into momentum space integrals according to (4.3). This is a common approach in the case of exponentially decaying basis functions (see for example [126] or [40] and references therein).

In the same way, we obtain the Fourier transform of the irregular solid harmonic, which again holds in the sense of distributions [126, Eq. (3.11)]:

$$
\begin{aligned}
\overline{\mathcal{Z}}_{\ell}^{m}(\boldsymbol{p}) & =(2 \pi)^{-3 / 2} \int \mathrm{e}^{-\mathrm{i} \boldsymbol{p} \cdot \boldsymbol{r}} \mathcal{Z}_{\ell}^{m}(\boldsymbol{r}) \mathrm{d}^{3} \boldsymbol{r} \\
& =(2 / \pi)^{1 / 2} \frac{(-1)^{\ell}}{(2 \ell-1) ! !} \frac{\mathcal{Y}_{\ell}^{m}(\mathrm{i} \boldsymbol{p})}{p^{2}} .
\end{aligned}
$$

It is also quite instructive to study the Fourier transforms of distributions as for instance the three-dimensional delta function or the spherical delta function. If we set $f\left(\boldsymbol{r}-\boldsymbol{r}^{\prime}\right)=\delta\left(\boldsymbol{r}-\boldsymbol{r}^{\prime}\right)$ in the convolution integral (4.1), we see that the Fourier transform of the delta function is a constant:

$$
\bar{\delta}(\boldsymbol{p})=(2 \pi)^{-3 / 2} \int \mathrm{e}^{-\mathrm{i} \boldsymbol{p} \cdot \boldsymbol{r}} \delta(\boldsymbol{r}) \mathrm{d}^{3} \boldsymbol{r}=(2 \pi)^{-3 / 2} .
$$

Similarly, we find that the Fourier transform of the spherical delta function is essentially a regular solid harmonic in momentum space:

$$
\begin{aligned}
\bar{\delta}_{\ell}^{m}(\boldsymbol{p}) & =(2 \pi)^{-3 / 2} \int \mathrm{e}^{-\mathrm{i} \boldsymbol{p} \cdot \boldsymbol{r}} \delta_{\ell}^{m}(\boldsymbol{r}) \mathrm{d}^{3} \boldsymbol{r} \\
& =(2 \pi)^{-3 / 2} \frac{(-1)^{\ell}}{(2 \ell-1) ! !} \mathcal{Y}_{\ell}^{m}(\mathrm{i} \boldsymbol{p}) .
\end{aligned}
$$

In physics, it is common to introduce the three-dimensional delta function via the Poisson equation (6.1). Both the Poisson equation (6.1) as well as its anisotropic generalization (6.3) follow from the Fourier transforms (6.4) and (6.5), respectively. We only have to take into account that the function $1 / p^{2}$, which occurs in both (6.4) and (6.5), is canceled by the Laplacian $\nabla^{2}$, whose Fourier transform is $-p^{2}$.

It is, however, just as well possible to introduce the there-dimensional delta function as well as the spherical delta function via the differential operator $1-\alpha^{-2} \nabla^{2}$ of the modified Helmholtz equation, whose Fourier transform

Ernst Joachim Weniger: The Spherical Tensor Gradient Operator 
is given by $\left[\alpha^{2}+p^{2}\right] / \alpha^{2}$. This follows at once from that fact that the Yukawa potential [140], which is an exponentially screened Coulomb potential with screening parameter $\alpha$, is also a special $B$ function according to

$$
\frac{\mathrm{e}^{-\alpha r}}{r}=\alpha \hat{k}_{-1 / 2}(\alpha r)=(4 \pi)^{1 / 2} \alpha B_{0,0}^{0}(\alpha, r) .
$$

Obviously, the Yukawa potential approaches the Coulomb potential in the limit of vanishing screening, i.e., for $\alpha \rightarrow 0$.

If we set $n=\ell=m=0$ in (5.6), we essentially obtain the Fourier transform of the Yukawa potential:

$$
\begin{aligned}
\bar{B}_{0,0}^{0}(\alpha, \boldsymbol{p}) & =(2 \pi)^{-3 / 2} \int \mathrm{e}^{-\mathrm{i} \boldsymbol{p} \cdot \boldsymbol{r}} B_{0,0}^{0}(\alpha, \boldsymbol{r}) \mathrm{d}^{3} \boldsymbol{r} \\
& =\left(2 \pi^{2}\right)^{-1 / 2} \frac{\alpha^{-1}}{\left[\alpha^{2}+p^{2}\right]} .
\end{aligned}
$$

Next, we use (4.1) to compute the convolution of a relatively arbitrary function $f: \mathbb{R}^{3} \rightarrow \mathbb{C}$ with the Yukawa potential [131, Eq. (6.9)]:

$$
\begin{gathered}
\int B_{0,0}^{0}\left(\alpha,\left[\boldsymbol{r}-\boldsymbol{r}^{\prime}\right]\right) f\left(\boldsymbol{r}^{\prime}\right) \mathrm{d} \boldsymbol{r}=\int \mathrm{e}^{\mathrm{i} \boldsymbol{r} \cdot \boldsymbol{p}} \bar{B}_{0,0}^{0}(\alpha, \boldsymbol{p}) \bar{f}(\boldsymbol{p}) \mathrm{d}^{3} \boldsymbol{p} \\
=\frac{\alpha^{-1}}{\left(2 \pi^{2}\right)^{1 / 2}} \int \frac{\mathrm{e}^{\mathrm{i} \boldsymbol{r} \cdot \boldsymbol{p}}}{\alpha^{2}+p^{2}} \bar{f}(\boldsymbol{p}) \mathrm{d}^{3} \boldsymbol{p} .
\end{gathered}
$$

If we now apply the differential operator $1-\alpha^{-2} \nabla^{2}$ to the convolution integral, interchange integration and differentiation, and use (5.10), we see that $B_{-1,0}^{0}(\alpha, \boldsymbol{r})=\left[1-\alpha^{-2} \nabla^{2}\right] B_{0,0}^{0}(\alpha, \boldsymbol{r})$ is proportional to the three-dimensional delta function [131, Eq. (6.10)]:

$$
\begin{aligned}
& {\left[1-\alpha^{-2} \nabla^{2}\right] \int B_{0,0}^{0}\left(\alpha,\left[\boldsymbol{r}-\boldsymbol{r}^{\prime}\right]\right) f\left(\boldsymbol{r}^{\prime}\right) \mathrm{d} \boldsymbol{r}} \\
& \quad=\int B_{-1,0}^{0}\left(\alpha,\left[\boldsymbol{r}-\boldsymbol{r}^{\prime}\right]\right) f\left(\boldsymbol{r}^{\prime}\right) \mathrm{d} \boldsymbol{r} \\
& \quad=\frac{(4 \pi)^{1 / 2}}{\alpha^{3}}(2 \pi)^{-3 / 2} \int \mathrm{e}^{\mathrm{i} \boldsymbol{r} \cdot \boldsymbol{p}} \bar{f}(\boldsymbol{p}) \mathrm{d}^{3} \boldsymbol{p}=\frac{(4 \pi)^{1 / 2}}{\alpha^{3}} f(\boldsymbol{r}) .
\end{aligned}
$$

We thus obtain the following exponentially screened variant of the Poisson equation (6.1):

$$
\left[1-\alpha^{-2} \nabla^{2}\right] B_{0,0}^{0}(\alpha, \boldsymbol{r})=\frac{(4 \pi)^{1 / 2}}{\alpha^{3}} \delta(\boldsymbol{r})
$$

Ernst Joachim Weniger: The Spherical Tensor Gradient Operator 
This relationship can also be expressed in terms of the Yukawa potential:

$$
\left[\alpha^{2}-\nabla^{2}\right] \frac{\mathrm{e}^{-\alpha r}}{r}=4 \pi \delta(\boldsymbol{r})
$$

For $\alpha=0$, we obtain the Poisson equation (6.1).

The screened Poisson equation (6.13) expresses the well known fact that the Yukawa potential is the Green's function of the modified Helmholtz equation (see for example [2, Table 16.1 on p. 912]).

An anisotropic generalization of the approach described above is also possible. If we set $n=-\ell$ in (5.9), we see that the application of the spherical tensor gradient operator to the Yukawa potential yields the socalled modified Helmholtz harmonic [133, Eq. (6.9)]:

$$
B_{-\ell, \ell}^{m}(\alpha, \boldsymbol{r})=(4 \pi)^{1 / 2}(-\alpha)^{-\ell} \mathcal{Y}_{\ell}^{m}(\nabla) B_{0,0}^{0}(\alpha, \boldsymbol{r})
$$

In the limit of vanishing screening, the modified Helmholtz harmonic approaches an irregular solid harmonic according to [126, Eq. (3.10)]

$$
\mathcal{Z}_{\ell}^{m}(\boldsymbol{r})=[(2 \ell-1) ! !]^{-1} \lim _{\alpha \rightarrow 0}\left[\alpha^{\ell+1} B_{-\ell, \ell}^{m}(\alpha, r)\right]
$$

If we set $n=-\ell$ in (5.6), we obtain the Fourier transform of the modified Helmholtz harmonic [129, Eq. (A.1)]:

$$
\bar{B}_{-\ell, \ell}^{m}(\alpha, \boldsymbol{p})=(2 / \pi)^{1 / 2} \frac{\alpha^{-\ell-1}}{\left[\alpha^{2}+p^{2}\right]} \mathcal{Y}_{\ell}^{m}(-i \boldsymbol{p})
$$

Both the Fourier integral producing this relationship as well as the inverse Fourier integral representation for $B_{-\ell, \ell}^{m}(\alpha, \boldsymbol{r})$ do not exist in the sense of classical analysis. In [129, Appendix] it was, however, shown that the classically divergent Fourier integral representation for $B_{-\ell, \ell}^{m}(\alpha, \boldsymbol{r})$ can be regularized by employing a suitable rational cutoff function.

If we now proceed as in the case of (6.11) and also use (4.4), we see that $B_{-\ell-1, \ell}^{m}(\alpha, \boldsymbol{r})=\left[1-\alpha^{-2} \nabla^{2}\right] B_{-\ell, \ell}^{m}(\alpha, \boldsymbol{r})$ is proportional to the spherical delta

Ernst Joachim Weniger: The Spherical Tensor Gradient Operator 
function [131, Eq. (6.17) - (6.19)]:

$$
\begin{aligned}
{\left[1-\alpha^{-2} \nabla^{2}\right] \int B_{-\ell, \ell}^{\ell}\left(\alpha,\left[\boldsymbol{r}-\boldsymbol{r}^{\prime}\right]\right) f\left(\boldsymbol{r}^{\prime}\right) \mathrm{d} \boldsymbol{r} } \\
\quad=\int B_{-\ell-1, \ell}^{\ell}\left(\alpha,\left[\boldsymbol{r}-\boldsymbol{r}^{\prime}\right]\right) f\left(\boldsymbol{r}^{\prime}\right) \mathrm{d} \boldsymbol{r} \\
\quad=\frac{(2 / \pi)^{1 / 2}}{\alpha^{\ell+3}} \int \mathrm{e}^{\mathrm{i} \boldsymbol{r} \cdot \boldsymbol{p}} \mathcal{Y}_{\ell}^{m}(-i \boldsymbol{p}) \bar{f}(\boldsymbol{p}) \mathrm{d}^{3} \boldsymbol{p} \\
\quad=(-1)^{\ell} \frac{4 \pi}{\alpha^{\ell+3}} \mathcal{Y}_{\ell}^{m}(\nabla) f(\boldsymbol{r}) .
\end{aligned}
$$

We thus obtain the following exponentially screened variant of the anisotropic Poisson equation (6.3):

$$
\begin{aligned}
& {\left[1-\alpha^{-2} \nabla^{2}\right] B_{-\ell, \ell}^{m}(\alpha, \boldsymbol{r})} \\
& \quad=(-1)^{\ell} \frac{4 \pi}{\alpha^{\ell+3}} \mathcal{Y}_{\ell}^{m}(\nabla) \delta(\boldsymbol{r})=\frac{4 \pi}{\alpha^{\ell+3}}(2 \ell-1) ! ! \delta_{\ell}^{m}(\boldsymbol{r}) .
\end{aligned}
$$

In view of this relationship and also because of (5.10) it makes sense to define a distributional $B$ function as the following derivative of the threedimensional Dirac delta function [131, Eq. (6.20)]:

$$
B_{-k-\ell, \ell}^{m}(\alpha, \boldsymbol{r})=\frac{(2 \ell-1) ! ! 4 \pi}{\alpha^{\ell+3}}\left[1-\alpha^{-2} \nabla^{2}\right]^{k-1} \delta_{\ell}^{m}(\boldsymbol{r}), \quad k \in \mathbb{N} .
$$

The fact that the distributional $B$ function $B_{-\ell-1, \ell}^{B}$ is proportional to the spherical delta function can also be seen by setting $n_{1}=-\ell_{1}-1$ in the convolution integral (5.7). Then, we obtain apart from a different prefactor the expression (5.12) for the product $\mathcal{Y}_{\ell_{1}}^{m_{1}}(\nabla) B_{n_{2}, \ell_{2}}^{m_{2}}(\alpha, \boldsymbol{r})$.

The distributional nature of some $B$ functions follows also from the fact that the Fourier transforms (5.6) of $B$ functions satisfy for all $n \in \mathbb{Z}$ and for all $n \in \mathbb{N}_{0}$ the following functional equations [131, Eqs. (6.21) - (6.23)]:

$$
\begin{aligned}
\bar{B}_{n, \ell}^{m}(\alpha, \boldsymbol{p}) & =\left[\alpha^{2} /\left(\alpha^{2}+p^{2}\right)\right] \bar{B}_{n-1, \ell}^{m}(\alpha, \boldsymbol{p}), \\
\bar{B}_{n, \ell}^{m}(\alpha, \boldsymbol{p}) & =\left[(4 \pi)^{1 / 2} / \alpha^{\ell}\right] \mathcal{Y}_{\ell}^{m}(-\mathrm{i} \boldsymbol{p}) \bar{B}_{n+\ell, 0}^{0}(\alpha, \boldsymbol{p}), \\
\bar{B}_{-1,0}^{0}(\alpha, \boldsymbol{p}) & =\alpha^{-3}\left(2 \pi^{2}\right)^{-1 / 2} .
\end{aligned}
$$

These functional equations in momentum space show that there is an intimate relationship between $B$ functions, the differential operator of the

Ernst Joachim Weniger: The Spherical Tensor Gradient Operator 
modified Helmholtz equation, the spherical tensor gradient operator, and the three-dimensional delta function.

There is a simple and intuitive interpretation of distributional $B$ functions. Because of the factorial $(n+\ell)$ ! in the denominator on the right-hand side of (5.5), $B$ functions are defined in the sense of classical analysis only if $n+\ell \geq 0$ holds. However, the definition of a $B$ function remains meaningful even for $n+\ell<0$. If $\boldsymbol{r} \neq \mathbf{0}$, the value of $B_{-k-\ell, \ell}^{m}(\boldsymbol{r})$ with $k \in \mathbb{N}$ is because of the singular factorial $(-k) !=\Gamma(-k+1)$ zero, but for $\boldsymbol{r}=\mathbf{0}$, its value is $\infty / \infty$ and therefore undefined. Thus, the mathematical nature of a $B$ function with $n+\ell<0$ as well as its value for $\boldsymbol{r}=\mathbf{0}$ has to be analyzed with the help of suitable limiting procedures.

\section{Addition Theorems}

In many subfields of physics and physical chemistry - for example in electrodynamics [53], in classical field theory [54, or in the theory of intermolecular forces 111 - an essential step towards a solution of the problem under consideration consists in a separation of variables.

Principal tools, which can accomplish such a separation of variables, are so-called addition theorems. These are expansions of a given function $f\left(\boldsymbol{r} \pm \boldsymbol{r}^{\prime}\right)$ with $\boldsymbol{r}, \boldsymbol{r}^{\prime} \in \mathbb{R}^{3}$ in products of other functions that only depend on either $\boldsymbol{r}$ or $\boldsymbol{r}^{\prime}$.

In view of the importance of addition theorems, it is not surprising that there is a very extensive literature. Consequently, any attempt to provide a reasonably complete bibliography would clearly be beyond the scope of this article. The interested reader is referred to the long, but nevertheless incomplete lists of references in [120, 122].

Addition theorems have played a major role in my own research. I first applied addition theorems for the evaluation of some multicenter integrals of $B$ functions in my Master's thesis [116, which was published in condensed form in [107. In my PhD thesis [117] and also afterwards, I preferred Fourier transformation for the evaluation of multicenter integrals of $B$ functions, but I later worked on the derivation of addition theorems [46, 118, 120, 122, 133, 136 .

In atomic or molecular calculations, we are predominantly interested in irreducible spherical tensors of the type of (2.2). Moreover, the convenient orthonormality of the spherical harmonics makes it highly desirable that the functions of either $\boldsymbol{r}$ or $\boldsymbol{r}^{\prime}$, which occur in the expansion of $f\left(\boldsymbol{r} \pm \boldsymbol{r}^{\prime}\right)$, are also

Ernst Joachim Weniger: The Spherical Tensor Gradient Operator 
irreducible spherical tensors. Thus, the addition theorems, we are interested in, are expansions in terms of spherical harmonics with arguments $\theta, \phi=\boldsymbol{r} / r$ and $\theta^{\prime}, \phi^{\prime}=\boldsymbol{r}^{\prime} / r^{\prime}$, respectively.

The best known example of such an addition theorem is the Laplace expansion of the Coulomb potential in terms of spherical harmonics:

$$
\begin{gathered}
\frac{1}{\left|\boldsymbol{r} \pm \boldsymbol{r}^{\prime}\right|}=4 \pi \sum_{\lambda=0}^{\infty} \frac{(\mp 1)^{\lambda}}{2 \lambda+1} \sum_{\mu=-\lambda}^{\lambda}\left[\mathcal{Y}_{\lambda}^{\mu}\left(\boldsymbol{r}_{<}\right)\right]^{*} \mathcal{Z}_{\lambda}^{\mu}\left(\boldsymbol{r}_{>}\right) \\
r_{<}=\min \left(r, r^{\prime}\right), \quad r_{>}=\max \left(r, r^{\prime}\right) .
\end{gathered}
$$

The Laplace expansion leads to a separation of the variables $\boldsymbol{r}$ and $\boldsymbol{r}^{\prime}$. However, its right-hand side depends on $\boldsymbol{r}$ and $\boldsymbol{r}^{\prime}$ only indirectly via the vectors $\boldsymbol{r}_{<}$and $\boldsymbol{r}_{>}$which satisfy $\left|\boldsymbol{r}_{<}\right|<\left|\boldsymbol{r}_{>}\right|$. Hence, the Laplace expansion has a two-range form, depending on the relative size of $\boldsymbol{r}$ and $\boldsymbol{r}^{\prime}$. This is a complication which occurs frequently among addition theorems. As discussed in more details in [120, 122, addition theorems have a two-range form if they are pointwise convergent three-dimensional Taylor expansions and if the function $f\left(\boldsymbol{r} \pm \boldsymbol{r}^{\prime}\right)$, which is to be expanded, is not analytic at the origin.

The undeniably troublesome two-range form of an addition theorem can be avoided if $f: \mathbb{R}^{3} \rightarrow \mathbb{C}$ belongs to the Hilbert space $L^{2}\left(\mathbb{R}^{3}\right)$ of square integrable functions or to other, closely related function spaces as for example Sobolev spaces that are proper subspaces of $L^{2}\left(\mathbb{R}^{3}\right)$ (compare for instance [11, 46, 118, 132] and references therein). For the sake of simplicity, let us assume that a discrete function set $\left\{\Psi_{n, \ell}^{m}(\boldsymbol{r})\right\}_{n, \ell, m}$ is complete and orthonormal in the Hilbert space $L^{2}\left(\mathbb{R}^{3}\right)$. Then, an addition theorem for $f\left(\boldsymbol{r} \pm \boldsymbol{r}^{\prime}\right)$, which converges in the mean with respect to the norm of $L^{2}\left(\mathbb{R}^{3}\right)$, can be derived by expanding $f$ in terms of the functions $\left\{\Psi_{n, \ell}^{m}(\boldsymbol{r})\right\}_{n, \ell, m}$ :

$$
\begin{aligned}
f\left(\boldsymbol{r} \pm \boldsymbol{r}^{\prime}\right) & =\sum_{n \ell m} C_{n, \ell}^{m}\left(f ; \boldsymbol{r}^{\prime}\right) \Psi_{n, \ell}^{m}(\boldsymbol{r}), \\
C_{n, \ell}^{m}\left(f ; \boldsymbol{r}^{\prime}\right) & =\int\left[\Psi_{n, \ell}^{m}(\boldsymbol{r})\right]^{*} f\left(\boldsymbol{r} \pm \boldsymbol{r}^{\prime}\right) \mathrm{d}^{3} \boldsymbol{r} .
\end{aligned}
$$

Such an expansion is a one-range addition theorem since the dependence on $\boldsymbol{r}$ is entirely contained in the functions $\Psi_{n, \ell}^{m}(\boldsymbol{r})$, whereas $\boldsymbol{r}^{\prime}$ occurs only in the expansion coefficients $C_{n, \ell}^{m}\left(f ; \boldsymbol{r}^{\prime}\right)$ which are overlap integrals. With minimal modifications, this approach works also if the functions $\left\{\Psi_{n, \ell}^{m}(\boldsymbol{r})\right\}_{n, \ell, m}$ are complete and orthonormal in a suitable Sobolev space.

Ernst Joachim Weniger: The Spherical Tensor Gradient Operator 
At first sight, it looks as if one-range addition theorems of the type of (7.2) are clearly superior to two-range addition theorems, but a balanced assessment of their relative merits is not so easy. Firstly, one-range addition theorems usually have a more complicated structure than two-range addition theorems (typically, one-range addition theorems contain one additional infinite summation). Secondly, one-range addition theorems normally converge only in the mean with respect to the norm of the Hilbert space $L^{2}\left(\mathbb{R}^{3}\right)$, but not necessarily pointwise. In some cases, this can lead to convergence problems. The probably the most severe disadvantage is that the approach sketched above works only if $f$ is an element of a suitable Hilbert or Sobolev space. Many functions of considerable practical importance such as the Coulomb potential or the irregular solid harmonic do not belong to $L^{2}\left(\mathbb{R}^{3}\right)$, let alone to a suitable Sobolev space. Therefore, it is not possible to derive one-range addition theorems by expanding them in terms of function sets that are complete and orthonormal with respect to a scalar product that involves an integration over the whole $\mathbb{R}^{3}$.

In this article, I will only discuss addition theorems that converge pointwise, i.e., which can be viewed to be three-dimensional Taylor expansions. Moreover, I will concentrate on addition theorems that can be derived with the help of the spherical tensor gradient operator $\mathcal{Y}_{\ell}^{m}(\nabla)$.

As discussed in Section [3] the irregular solid harmonic $\mathcal{Z}_{\ell}^{m}(\boldsymbol{r})$ can according to (3.9) be obtained by applying $\mathcal{Y}_{\ell}^{m}(\nabla)$ to the Coulomb potential. Thus, it should be possible to derive the addition theorem of the irregular solid harmonic by applying either $\mathcal{Y}_{\ell}^{m}\left(\nabla_{<}\right)$or $\mathcal{Y}_{\ell}^{m}\left(\nabla_{>}\right)$to the Laplace expansion (7.1). As shown in [133, Section IV], this is indeed possible. Moreover, it was shown in [133, Sections V and VI] that the addition theorems of the Helmholtz and the modified Helmholtz harmonics can also be derived by applying either $\mathcal{Y}_{\ell}^{m}\left(\nabla_{<}\right)$or $\mathcal{Y}_{\ell}^{m}\left(\nabla_{>}\right)$to the simpler addition theorems of the corresponding scalar functions. It is also possible to derive the addition theorem of $B$ functions by applying the spherical tensor gradient operator to the addition theorem of the reduced Bessel functions [136, Section V].

The approach described above requires that for a given irreducible tensor a scalar function can be found which satisfies (3.5) and which possesses a suitable addition theorem. This obviously limits the practical usefulness of this approach. It is, however, possible to derive addition theorems of essentially arbitrary irreducible spherical tensors from the scratch with the help of the spherical tensor gradient operator.

As is well known, addition theorems can formally be obtained by doing

Ernst Joachim Weniger: The Spherical Tensor Gradient Operator 
three-dimensional Taylor expansions (see for example [12 p. 181]):

$$
f\left(\boldsymbol{r}+\boldsymbol{r}^{\prime}\right)=\sum_{n=0}^{\infty} \frac{\left(\boldsymbol{r}^{\prime} \cdot \nabla\right)^{n}}{n !} f(\boldsymbol{r})=\mathrm{e}^{\boldsymbol{r}^{\prime} \cdot \nabla} f(\boldsymbol{r}) .
$$

Thus, the translation operator

$$
\mathrm{e}^{\boldsymbol{r}^{\prime} \cdot \nabla}=\mathrm{e}^{x^{\prime} \partial / \partial x} \mathrm{e}^{y^{\prime} \partial / \partial y} \mathrm{e}^{z^{\prime} \partial / \partial z}
$$

generates $f\left(\boldsymbol{r}+\boldsymbol{r}^{\prime}\right)$ by doing a three-dimensional Taylor expansion of $f$ around $\boldsymbol{r}$ with shift vector $\boldsymbol{r}^{\prime}$. Moreover, the variables $\boldsymbol{r}$ and $\boldsymbol{r}^{\prime}$ are separated. Thus, the series expansion (7.3) is indeed an addition theorem.

We could also expand $f$ around $\boldsymbol{r}^{\prime}$ and use $\boldsymbol{r}$ as the shift vector. This would produce an addition theorem for $f\left(\boldsymbol{r}+\boldsymbol{r}^{\prime}\right)$ in which the roles of $\boldsymbol{r}$ and $\boldsymbol{r}^{\prime}$ are interchanged. Both approaches are mathematically legitimate and equivalent if $f$ is analytic at $\boldsymbol{r}, \boldsymbol{r}^{\prime}$, and $\boldsymbol{r}+\boldsymbol{r}^{\prime}$ for essentially arbitrary vectors $\boldsymbol{r}, \boldsymbol{r}^{\prime} \in \mathbb{R}^{3}$. Unfortunately, this is normally not true. Most functions, that are of interest in the context of atomic and molecular quantum mechanics, are either singular at the origin or are not analytic at the origin. Obvious examples are the Coulomb potential, which is singular at the origin, or the $1 \mathrm{~s}$ hydrogen eigenfunction, which possesses a cusp at the origin. In fact, all the commonly used exponentially decaying functions as for example Slater-type functions or also $B$ functions are not analytic at the origin.

The reason for the non-analyticity is that the three-dimensional distance $r=\left[x^{2}+y^{2}+z^{2}\right]^{1 / 2}$ is not analytic with respect to $x, y$, and $z$ at the origin $\boldsymbol{r}=\mathbf{0}$. This implies that all odd powers $r^{2 n+1}$ with $n \in \mathbb{N}_{0}$ are also not analytic at the origin (compare also the discussion related to [118. Eq. (5.9)]). In contrast, $r^{2}=x^{2}+y^{2}+z^{2}$ and the regular solid harmonic $\mathcal{Y}_{\ell}^{m}(\boldsymbol{r})$ are analytic since they are polynomials in $x, y$, and $z$. Consequently, a $1 s$ Gaussian function $\exp \left(-\alpha r^{2}\right)$, which possesses an expansion in even powers $r^{2 n}$, is analytic at $\boldsymbol{r}=\mathbf{0}$, but a $1 s$ Slater-type function $\exp (-\alpha r)$ is not.

Thus, for the derivation of addition theorems for functions, that are not analytic at the origin, we have to use the translation operator in the following form,

$$
f\left(\boldsymbol{r}_{<}+\boldsymbol{r}_{>}\right)=\sum_{n=0}^{\infty} \frac{\left(\boldsymbol{r}_{<} \cdot \nabla_{>}\right)^{n}}{n !} f\left(\boldsymbol{r}_{>}\right)=\mathrm{e}^{\boldsymbol{r}_{<} \cdot \nabla_{>}} f\left(\boldsymbol{r}_{>}\right),
$$

where $\left|\boldsymbol{r}_{<}\right|<\left|\boldsymbol{r}_{>}\right|$. In this way, the convergence of the three-dimensional Taylor expansion is guaranteed provided that $f$ is analytic everywhere except

Ernst Joachim Weniger: The Spherical Tensor Gradient Operator 
possibly at the origin. Thus, the non-analyticity of $B$ functions and of all the other commonly occurring exponentially decaying functions at the origin has a far-reaching consequence: Their pointwise convergent addition theorems must have a two-range form in order to guarantee convergence (see also 122 and references therein).

From a practical point of view, the translation operator $\mathrm{e}^{\boldsymbol{r}_{<} \cdot \nabla>}$ in its Cartesian form does not seem to be a particularly useful analytical tool. We are usually interested in addition theorems of irreducible spherical tensors, which are defined in terms of the spherical polar coordinates $r, \theta$, and $\phi$. Differentiating them with respect to the Cartesian components $x, y$, and $z$ would lead to extremely messy expressions and to difficult technical problems. Thus, it is tempting, but nevertheless superficial to conclude that the translation operator $\mathrm{e}^{\boldsymbol{r}_{<} \cdot \nabla_{>}}$provides only a formal solution to the problem of separating the variables $\boldsymbol{r}$ and $\boldsymbol{r}^{\prime}$ of a function $f\left(\boldsymbol{r}+\boldsymbol{r}^{\prime}\right)$.

The crucial step, which ultimately makes the Taylor expansion method practically useful, is the expansion of the translation operator $\mathrm{e}^{\boldsymbol{r}_{<} \cdot \nabla>}$ in terms of differential operators that are irreducible spherical tensors. The starting point is an expansion of $\exp (\boldsymbol{a} \cdot \boldsymbol{b})$ with $\boldsymbol{a}, \boldsymbol{b} \in \mathbb{R}^{3}$ in terms of modified Bessel functions and Legendre polynomials [61, p. 108]:

$$
\mathrm{e}^{\boldsymbol{a} \cdot \boldsymbol{b}}=\mathrm{e}^{a b \cos \theta}=\left(\frac{\pi}{2 a b}\right)^{1 / 2} \sum_{\ell=0}^{\infty}(2 \ell+1) I_{\ell+1 / 2}(a b) P_{\ell}(\cos \theta) .
$$

Next, the series expansion for the modified Bessel function $I_{\ell+1 / 2}$ [61, p. $66]$ is inserted into (7.6), and spherical harmonics are introduced with the help of the so-called addition theorem of the Legendre polynomials (see for example [12, p. 303]). This yields the following expansion of $\mathrm{e}^{\boldsymbol{a} \cdot \boldsymbol{b}}$ in terms of regular solid harmonics and even powers of the vectors $\boldsymbol{a}$ and $\boldsymbol{b}$ :

$$
\mathrm{e}^{\boldsymbol{a} \cdot \boldsymbol{b}}=2 \pi \sum_{\ell=0}^{\infty} \sum_{m=-\ell}^{\ell}\left[\mathcal{Y}_{\ell}^{m}(\boldsymbol{a})\right]^{*} \mathcal{Y}_{\ell}^{m}(\boldsymbol{b}) \sum_{k=0}^{\infty} \frac{\boldsymbol{a}^{2 k} \boldsymbol{b}^{2 k}}{2^{\ell+2 k} k !(1 / 2)_{\ell+k+1}}
$$

The powers $\boldsymbol{a}^{2 k}$ and $\boldsymbol{b}^{2 k}$ are irreducible spherical tensors of rank zero, and the solid harmonics are tensors of rank $\ell$.

The expansion (7.7) is obtained from (17.6) by rearranging the Cartesian components of the vectors $\boldsymbol{a}$ and $\boldsymbol{b}$. Accordingly, it holds for essentially

Ernst Joachim Weniger: The Spherical Tensor Gradient Operator 
arbitrary vectors $\boldsymbol{a}, \boldsymbol{b} \in \mathbb{R}^{3}$, and we can choose $\boldsymbol{a}=\boldsymbol{r}_{<}$and $\boldsymbol{b}=\nabla_{>}$:

$$
\mathrm{e}^{\boldsymbol{r}_{<} \cdot \nabla>}=2 \pi \sum_{\ell=0}^{\infty} \sum_{m=-\ell}^{\ell}\left[\mathcal{Y}_{\ell}^{m}\left(\boldsymbol{r}_{<}\right)\right]^{*} \mathcal{Y}_{\ell}^{m}\left(\nabla_{>}\right) \sum_{k=0}^{\infty} \frac{\boldsymbol{r}_{<}^{2 k} \nabla_{>}^{2 k}}{2^{\ell+2 k} k !(1 / 2)_{\ell+k+1}} .
$$

It seems that this expansion was first published by Santos [95. Eq. (A.6)], who emphasized that this expansion should be useful for the derivation of addition theorems, but apparently never used it for that purpose.

It follows from the expression (B.2) of the Laplacian $\nabla^{2}$ in spherical polar coordinates and from the tensorial nature of the spherical tensor gradient operator (compare (2.3) and the numerous expressions for $\gamma_{\ell_{1} \ell_{2}}^{\ell}(r)$ given in Section 4) that in (7.8) we only have to do differentiations with respect to the radial variable $r_{>}$. This greatly simplifies practical applications. In [120, it was shown that starting from the expansion (7.8) the addition theorems of the Coulomb potential, the regular and irregular solid harmonics, and the Yukawa potential can be derived quite easily, and in [122], several different forms of the addition theorem of the $B$ functions was derived in this way.

As a further demonstration of the usefulness of the expansion (7.8) of the translation operator in terms of irreducible spherical tensors I will now derive an addition theorem of the function $r^{\nu} \mathcal{Y}_{\ell}^{m}(\boldsymbol{r})$ with $\nu \in \mathbb{R}$.

Our starting point the the following relationship which follows at once from (3.4):

$$
r^{\nu} \mathcal{Y}_{\ell}^{m}(\boldsymbol{r})=\frac{1}{2^{\ell}(1+\nu / 2)_{\ell}} \mathcal{Y}_{\ell}^{m}(\nabla) r^{\nu+2 \ell} .
$$

If we combine this with (7.8) and linearize the spherical tensor gradient operators according to (3.7), we obtain:

$$
\begin{aligned}
& \mid \boldsymbol{r}_{<}+\left.\boldsymbol{r}_{>}\right|^{\nu} \mathcal{Y}_{\ell}^{m}\left(\boldsymbol{r}_{<}+\boldsymbol{r}_{>}\right)=\frac{2 \pi}{2^{\ell}(1+\nu / 2)_{\ell}} \sum_{\ell_{1}=0}^{\infty} \sum_{m_{1}=-\ell_{1}}^{\ell_{1}}\left[\mathcal{Y}_{\ell_{1}}^{m_{1}}\left(\boldsymbol{r}_{<}\right)\right]^{*} \\
& \quad \times \sum_{\ell_{2}=\ell_{2}^{\min }}^{(2)}\left\langle\ell_{2} m+m_{1}\left|\ell_{1} m_{1}\right| \ell m\right\rangle \sum_{k=0}^{\infty} \frac{r_{<}^{2 k}}{2^{\max }} \\
& \quad \times \nabla_{>}^{2 k+2 \Delta \ell_{2}} \mathcal{Y}_{\ell_{2}}^{m+m_{1}}\left(\nabla_{>}\right) r_{>}^{\nu+2 \ell} .
\end{aligned}
$$

The abbreviations $\Delta \ell, \Delta \ell_{1}$, and $\Delta \ell_{2}$, which will be used in the following formulas and which are in all cases either zero or a positive integer, are defined by (C.6) - (C.8),

Ernst Joachim Weniger: The Spherical Tensor Gradient Operator 
In the next step, we use (7.8) once more to obtain

$$
\mathcal{Y}_{\ell_{2}}^{m+m_{1}}(\nabla) r^{\nu+2 \ell}=(-2)^{\ell_{2}}(-\ell-\nu / 2)_{\ell_{2}} r^{\nu+2 \ell-2 \ell_{2}} \mathcal{Y}_{\ell_{2}}^{m+m_{1}}(\boldsymbol{r}) \text {. }
$$

Inserting this into (7.10) yields:

$$
\begin{aligned}
\left|\boldsymbol{r}_{<}+\boldsymbol{r}_{>}\right|^{\nu} \mathcal{Y}_{\ell}^{m}\left(\boldsymbol{r}_{<}+\boldsymbol{r}_{>}\right)=\frac{2 \pi}{2^{\ell}(1+\nu / 2)_{\ell}} \sum_{\ell_{1}=0}^{\infty} \sum_{m_{1}=-\ell_{1}}^{\ell_{1}}\left[\mathcal{Y}_{\ell_{1}}^{m_{1}}\left(\boldsymbol{r}_{<}\right)\right]^{*} \\
\times \sum_{\ell_{2}=\ell_{2}^{\min }}^{(2)}(-1)^{\ell_{2}}\left\langle\ell_{2} m+m_{1}\left|\ell_{1} m_{1}\right| \ell m\right\rangle \\
\quad \times \sum_{k=0}^{\infty} \frac{2^{\ell_{2}-\ell_{1}-2 k}(-\ell-\nu / 2)_{\ell_{2}} r_{<}^{2 k}}{k !(1 / 2)_{\ell_{1}+k+1}} \\
\times \nabla_{>}^{2 k+2 \Delta \ell_{2}} r_{>}^{\nu+2 \ell-2 \ell_{2}} \mathcal{Y}_{\ell_{2}}^{m+m_{1}}\left(\boldsymbol{r}_{>}\right) .
\end{aligned}
$$

Now, the only thing, that remains to be done, is the application of the powers $\nabla_{>}^{2 k+2 \Delta \ell_{2}}$ of the Laplacian to $r_{>}^{\nu+2 \ell-2 \ell_{2}} \mathcal{Y}_{\ell_{2}}^{m+m_{1}}\left(\boldsymbol{r}_{>}\right)$. For that purpose, we use the following expression which can be deduced easily from (B.2):

$$
\nabla^{2} \psi(r) \mathcal{Y}_{\ell}^{m}(\boldsymbol{r})=\mathcal{Y}_{\ell}^{m}(\boldsymbol{r})\left[\frac{\partial^{2}}{\partial r^{2}}+\frac{2 \ell+2}{r} \frac{\partial}{\partial r}\right] \psi(r) .
$$

Here, $\psi(r)$ is a scalar function. If we set $\psi(r)=r^{\sigma}$ with $\sigma \in \mathbb{R}$, we find:

$$
\nabla^{2} r^{\sigma} \mathcal{Y}_{\ell}^{m}(\boldsymbol{r})=4(-\sigma / 2)(-[\sigma+2 \ell+1] / 2) r^{\sigma-2} \mathcal{Y}_{\ell}^{m}(\boldsymbol{r}) .
$$

Iterating this relationship $n$ times yields:

$$
\nabla^{2 n} r^{\sigma} \mathcal{Y}_{\ell}^{m}(\boldsymbol{r})=4^{n}(-\sigma / 2)_{n}(-[\sigma+2 \ell+1] / 2)_{n} r^{\sigma-2 n} \mathcal{Y}_{\ell}^{m}(\boldsymbol{r}) .
$$

Thus, we obtain for the remaining differentiations:

$$
\begin{aligned}
& \nabla_{>}^{2 k+2 \Delta \ell_{2}} r_{>}^{\nu+2 \ell-2 \ell_{2}} \mathcal{Y}_{\ell_{2}}^{m+m_{1}}\left(\boldsymbol{r}_{>}\right)=4^{k+\Delta \ell_{2}} \\
& \quad \times\left(-\frac{\nu+2 \ell-2 \ell_{2}}{2}\right)_{\Delta \ell_{2}}\left(-\frac{\nu+2 \ell+1}{2}\right)_{\Delta \ell_{2}}\left(\frac{2 \Delta \ell-\nu}{2}\right)_{k} \\
& \quad \times\left(-\frac{2 \Delta \ell_{1}+\nu+1}{2}\right)_{k} r_{>}^{\nu+2 \Delta \ell_{1}-2 k+1} \mathcal{Z}_{\ell_{2}}^{m+m_{1}}\left(\boldsymbol{r}_{>}\right) .
\end{aligned}
$$

\section{Ernst Joachim Weniger: The Spherical Tensor Gradient Operator}


With the help of some essentially straightforward algebra, it can be shown that the $k$ summation in (17.12) can be expression by a Gaussian hypergeometric series ${ }_{2} F_{1}$ (for its definition, see for instance [61, p. 37]), and we finally obtain the following addition theorem:

$$
\begin{gathered}
\left|\boldsymbol{r}_{<}+\boldsymbol{r}_{>}\right|^{\nu} \mathcal{Y}_{\ell}^{m}\left(\boldsymbol{r}_{<}+\boldsymbol{r}_{>}\right)=\frac{4 \pi}{(1+\nu / 2)_{\ell}} \sum_{\ell_{1}=0}^{\infty} \sum_{m_{1}=-\ell_{1}}^{\ell_{1}}\left[\mathcal{Y}_{\ell_{1}}^{m_{1}}\left(\boldsymbol{r}_{<}\right)\right]^{*} \\
\times \sum_{\ell_{2}=\ell_{2}^{\min }}^{\ell_{2}^{\max }}(-1)^{\ell_{2}}\left\langle\ell_{2} m+m_{1}\left|\ell_{1} m_{1}\right| \ell m\right\rangle \\
\times \frac{(-\ell-\nu / 2)_{\ell_{2}}}{(3 / 2)_{\ell_{1}}}\left(\frac{\nu-2 \Delta \ell+2}{2}\right)_{\Delta \ell_{2}}\left(\frac{\nu-2 \Delta \ell+3}{2}\right)_{\Delta \ell_{2}} \\
\times{ }_{2} F_{1}\left(\frac{2 \Delta \ell-\nu}{2}, \frac{-2 \Delta \ell_{1}-\nu-1}{2} ; \frac{2 \ell_{1}+3}{2} ; \frac{r_{<}^{2}}{r_{>}^{2}}\right) \\
\times r_{>}^{\nu+2 \Delta \ell_{1}+1} \mathcal{Z}_{\ell_{2}}^{m+m_{1}}\left(\boldsymbol{r}_{>}\right) .
\end{gathered}
$$

The addition theorem (7.17) contains many simpler addition theorems as special cases. For example, if we set in (7.17) $\ell=m=0$, we obtain the addition theorem of the corresponding scalar function:

$$
\begin{array}{rl}
\left|\boldsymbol{r}_{<}+\boldsymbol{r}_{>}\right|^{\nu}=4 & 4 r_{>}^{\nu+1} \sum_{\lambda=0}^{\infty}(-1)^{\lambda} \frac{(-\nu / 2)_{\lambda}}{(3 / 2)_{\lambda}} \\
\times{ }_{2} F_{1}\left(\frac{2 \lambda-\nu}{2}, \frac{-\nu-1}{2} ; \frac{2 \lambda+3}{2} ; \frac{r_{<}^{2}}{r_{>}^{2}}\right) \\
\times \sum_{\mu=-\lambda}^{\lambda}\left[\mathcal{Y}_{\lambda}^{\mu}\left(\boldsymbol{r}_{<}\right)\right]^{*} \mathcal{Z}_{\lambda}^{\mu}\left(\boldsymbol{r}_{>}\right) .
\end{array}
$$

Of course, we could also go the other way round: We could proceed as in 133] and construct the addition theorem (17.17) by applying either $\mathcal{Y}_{\ell}^{m}\left(\nabla_{<}\right)$ or $\mathcal{Y}_{\ell}^{m}\left(\nabla_{>}\right)$to (7.18).

If we set in (7.18) $\nu=-1$, we only need $(1 / 2)_{\lambda} /(3 / 2)_{\lambda}=1 /(2 \lambda+1)$ as well as ${ }_{2} F_{1}\left(\lambda+1 / 2,0 ; \lambda+3 / 2 ; r_{<}^{2} / r_{>}^{2}\right)=1$ to obtain the Laplace expansion (17.1) of the Coulomb potential.

If we set in (7.18) $\nu=2 n$ with $n \in \mathbb{N}_{0}$, the $\lambda$ summation terminates after $\lambda=n$ because of the Pochhammer symbol $(-n)_{\lambda}$. In addition, the

Ernst Joachim Weniger: The Spherical Tensor Gradient Operator 
hypergeometric series ${ }_{2} F_{1}$ in (7.18) terminates to become a polynomial of degree $n-\lambda$ in $r_{<}^{2} / r_{>}^{2}$. Moreover, $r^{2 n}$ is a polynomial in $x, y, z$ and thus analytic. Accordingly, a distinction between $\boldsymbol{r}_{<}$and $\boldsymbol{r}_{>}$is not necessary and the addition theorem has a one-range form.

A detailed analysis of all special cases of the addition theorem (7.17) would clearly be beyond the scope of this article (see also [114, pp. 168169]). Here, one must not forget that the emphasis of this Section is not on the derivation of the addition theorem (7.17). Rather, it is my hope that the fairly effortless derivation of this addition theorem convinces the reader that the expansion (7.8) of the translation operator in terms of irreducible spherical tensors is indeed a highly useful analytical tool. Of course, a sceptical reader may well argue that it is unlikely that convenient explicit expression for the necessary radial differentiations of arbitrary order can always be found, as it was the case with (7.17). This certainly true. But even if we can only do the differentiations explicitly up to a finite maximum order, the tensorial expansion (7.8) permits at least the construction of approximations to addition theorems. Computer algebra systems like Maple or Mathematica should be helpful in this respect.

\section{Summary and Conclusions}

The regular solid harmonic $\mathcal{Y}_{\ell}^{m}(\boldsymbol{r})=r^{\ell} Y_{\ell}^{m}(\theta, \phi)$ is according to (B.12) a homogeneous polynomial of degree $\ell$ in the Cartesian components of $\boldsymbol{r}$. Thus, it makes sense to define the differential operator $\mathcal{Y}_{\ell}^{m}(\nabla)$ via (2.1), i.e., by replacing the Cartesian components of $\boldsymbol{r}$ in (B.12) by the Cartesian components of $\nabla$.

The spherical tensor gradient operator $\mathcal{Y}_{\ell}^{m}(\nabla)$ is an irreducible spherical tensor of rank $\ell$. This is a very consequential fact. Firstly, its application to a scalar function, which is an irreducible spherical tensor of rank 0 , must produce an irreducible spherical tensor of rank $\ell$. This follows at once from the simplified version (3.4) of Hobson's theorem which is discussed in Section 3. With the help of Hobson's theorem, it is also possible to derive the compact explicit expression (3.8) for the product $\mathcal{Y}_{\ell_{1}}^{m_{1}}(\nabla) F_{\ell_{2}}^{m_{2}}(\boldsymbol{r})$, where $F_{\ell_{2}}^{m_{2}}$ is an irreducible spherical tensor with nonzero rank $\ell_{2}$ of the type of (2.2) that also satisfies (3.4). Secondly, the structure of products of the type of $\mathcal{Y}_{\ell_{1}}^{m_{1}}(\nabla) F_{\ell_{2}}^{m_{2}}(\boldsymbol{r})$ according to (2.3) can be understood completely on the basis of the usual coupling rules of angular momentum theory. Accordingly, the angular parts of such a product can be expressed in terms of Gaunt coeffi-

Ernst Joachim Weniger: The Spherical Tensor Gradient Operator 
cients and spherical harmonics. Moreover, the radial parts of $\mathcal{Y}_{\ell_{1}}^{m_{1}}(\nabla) F_{\ell_{2}}^{m_{2}}(\boldsymbol{r})$ and of $F_{\ell_{2}}^{m_{2}}(\boldsymbol{r})$ are connected by relationships which only involve differentiations with respect to the radial variable $r$. It is this property which makes the spherical tensor gradient operator a practically useful analytical tool.

Fourier transformation is one of the principal techniques for the evaluation of molecular multicenter integrals. It is also extremely useful in connection with the spherical tensor gradient operator. Under Fourier transformation, the differential operator $\mathcal{Y}_{\ell_{1}}^{m_{2}}(\nabla)$ produces a regular solid harmonic $\mathcal{Y}_{\ell_{1}}^{m_{2}}(\mathrm{i} \boldsymbol{p})$ in momentum space. In this way, we can easily understand the tensorial nature of $\mathcal{Y}_{\ell_{1}}^{m_{2}}(\nabla)$. Moreover, many analytical manipulations can be done more conveniently in the momentum than in the coordinate representation.

There are some scalar functions of physical interest which produce particularly compact results if $\mathcal{Y}_{\ell_{1}}^{m_{2}}(\nabla)$ is applied to them. Examples are the Coulomb potential, which produces an irregular solid harmonic according to (3.9), or the $1 s$ Gaussian function, which produces a spherical Gaussian function according to (3.11). Another class of scalar functions, to which $\mathcal{Y}_{\ell_{1}}^{m_{1}}(\nabla)$ can be applied with remarkable ease, are the so-called reduced Bessel functions defined in (5.1), and their anisotropic generalizations, the so-called $B$ functions defined in (5.5). These functions, which have played a major role in my own research, are discussed in Section 5. Application of the spherical tensor gradient operator to a scalar $B$ function simply produces a nonscalar $B$ function according to (5.9), and the product $\mathcal{Y}_{\ell_{1}}^{m_{1}}(\nabla) B_{n_{2}, \ell_{2}}^{m_{2}}(\alpha, \boldsymbol{r})$ can according to (5.12) be expressed by a simple linear combination of $B$ functions. The simplicity of both (5.9) and (5.12) follows directly from the very simple Fourier transform (5.6) of $B$ functions, which gives them an exceptional position among exponentially decaying basis functions.

Classically, the domain of the spherical tensor gradient operator consists of the differentiable functions $f: \mathbb{R}^{3} \rightarrow \mathbb{C}$. However, as discussed in Section [6] it makes sense to apply $\mathcal{Y}_{\ell}^{m}(\nabla)$ also to distribution or generalized functions. This produces mathematical objects like the spherical delta function $\delta_{\ell}^{m}(\boldsymbol{r})$, which is defined by (6.2) and which can be viewed as a generalized solution of the Poisson equation (6.3) of a unit multipole charge. With the help of Fourier transformation, the nature of the spherical delta function can be made transparent, and we can also understand easily why the Poisson equation (6.1) holds, or - to put it differently - why convolution with the Coulomb potential and application of the Laplace operator are inverse operations. An essentially identical approach works also in the case of $B$

Ernst Joachim Weniger: The Spherical Tensor Gradient Operator 
functions. It follows from (5.10) or also from the functional equations (6.20) that the differential operator $1-\alpha^{-2} \nabla^{2}$ of the modified Helmholtz equation functions can be viewed as a kind of lowering operator for $B$ functions. Since the Yukawa potential $\exp (-\alpha r) / r$ is according to (6.8) proportional to the $B$ function $B_{0,0}^{0}(\alpha, \boldsymbol{r})$, it turns out that convolution with the Yukawa potential and the application of the differential operator $1-\alpha^{-2} \nabla^{2}$ are according to (6.11) inverse operations.

In many subfields of physics and physical chemistry, an essential step towards a solution of the problem under consideration consists in a separation of variables. Addition theorems, which are discussed in Section 7. are principal tools to accomplish such a separation of variables. As is well known, addition theorems can be obtained according to (17.3) by applying the translation operator $\mathrm{e}^{\boldsymbol{r}^{\prime} \cdot \nabla}$ to a function $f(\boldsymbol{r})$. However, in atomic and molecular electronic structure calculations, we are predominantly interested in irreducible spherical tensors of the type of (2.2), and the convenient orthonormality of the spherical harmonics makes it highly desirable that the functions, which occur in the addition theorem, are also irreducible spherical tensors of the type of (2.2). Accordingly, the translation operator in its Cartesian form (7.4) is not suited for our needs, since its use would lead to enormous technical problems. It is a much better idea to express the translation operator in terms of irreducible spherical tensors according to (7.8). The feasibility of this approach is demonstrated by deriving the addition theorem of the function $r^{\nu} \mathcal{Y}_{\ell}^{m}(\boldsymbol{r})$ with $\nu \in \mathbb{R}$.

Let me conclude this article by some personal remarks. There can be no doubt that $\mathcal{Y}_{\ell}^{m}(\nabla)$ is a useful analytical tool since its application to a scalar function produces an irreducible spherical tensor of rank $\ell$. This is a highly advantageous feature, because now it is in principle sufficient to consider only multicenter integrals of scalar functions. Higher angular momentum states can be generated by differentiation with respect to the nuclear coordinates. Often, this is simpler that the direct derivation of explicit expressions for integrals of nonscalar functions. This approach has the additional advantage that it facilitates the use of computer algebra systems like Maple or Mathematica, whose systematic utilization I wholeheartedly recommend. The completely symbolic treatment of complicated multicenter integrals is still too difficult for computer algebra systems, but they are already now very well suited for doing complicated differentiations.

Nevertheless, one should never forget that a nice explicit expression for a multicenter integral does not necessarily permit its efficient and reliable eval-

Ernst Joachim Weniger: The Spherical Tensor Gradient Operator 
uation. Already during the process of deriving an explicit expression, one should always take numerical aspects into account, because they ultimately decide whether a given formula is practically useful or not. Unfortunately, this is more easily said than done. Thus, the ability of skillfully manipulating complicated mathematical expressions does not guarantee success in the integral business. It is also necessary to have a detailed knowledge of numerical analysis.

For example, during the work for my $\mathrm{PhD}$ thesis [117, series expansions for multicenter integrals played a major role. Unfortunately, it often happened that series expansions converged quite slowly (see for example [130. Table II]). In principle, it is a fairly obvious idea to try to speed up the convergence of these expansions with the help of series transformations, but during my PhD thesis I only knew the linear series transformations described in the classic book by Knopp [57, which did not accomplish much. At that time, I was completely ignorant about the more modern and more effective computational tools such as Padé approximants or other nonlinear transformations, which often accomplish spectacular improvements of convergence.

The situation changed radically when I did postdoctoral work with Professor Jiří Cížek at the Department of Applied Mathematics of the University of Waterloo. There, I worked on distributive expansions of a plane wave 118, which converge weakly with respect to the norm of the Hilbert space $L^{2}\left(\mathbb{R}^{3}\right)$ or the Sobolev space $W_{2}^{(1)}\left(\mathbb{R}^{3}\right)$. My second research topic in Waterloo - the summation of factorially divergent power series as they for instance occur as asymptotic expansions for special functions or in perturbation expansions of quantum physics - was in the long run far more consequential, although I did not accomplish anything worth publishing during my stay in 1983.

After my return to Regensburg, I tried to apply nonlinear transformations also to slowly convergent series expansions for multicenter integrals. In some cases, remarkable improvements of convergence were observed 41, 108, 125, 134, 135.

In order to understand better the power as well as the limitations of nonlinear sequence transformations, I also worked on their theoretical properties. As a by-product, I was able to derive several new transformations. The majority of these transformations was published in my long article [119, where also efficient algorithms for the computation of sequence transformations are discussed as well as theoretical error estimates and convergence properties. More recent activities are described in the articles [7, 121, 123, 124, 127.

Ernst Joachim Weniger: The Spherical Tensor Gradient Operator 
Thus, it is probably justified to claim that numerical mathematics ultimately profited via cross fertilizations from the convergence problems which I encountered. My personal experience highlights the central importance of a functioning communication between mathematicians and scientists. Generalists like Professor Josef Paldus, who is at home both in mathematics and in physical and theoretical chemistry, help to overcome the current communication problems and thus do an invaluable service to the scientific community.

\section{A Appendix: Terminology and Definitions}

For the set of positive and negative integers, I write $\mathbb{Z}=\{0, \pm 1, \pm 2, \ldots\}$, for the set of positive integers, I write $\mathbb{N}=\{1,2,3, \ldots\}$, and for the set of nonnegative integers, I write $\mathbb{N}_{0}=\{0,1,2, \ldots\}$. The real and complex numbers and the set of three-dimensional vectors with real components are denoted by $\mathbb{R}, \mathbb{C}$, and $\mathbb{R}^{3}$, respectively.

For the commonly occurring special functions of mathematical physics I use the notation of Magnus, Oberhettinger, and Soni 61 unless explicitly stated otherwise.

Double factorials are defined according to

$$
\begin{aligned}
(2 n) ! ! & =2 \cdot 4 \cdots 2 n, \quad n \in \mathbb{N}, \\
(2 n-1) ! ! & =1 \cdot 3 \cdots(2 n-1), \quad n \in \mathbb{N}, \\
0 ! ! & =1 ! !=(-1) ! !=1 .
\end{aligned}
$$

Fourier transformation is used used in its symmetrical form, i.e., a function $f: \mathbb{R}^{3} \rightarrow \mathbb{C}$ and its Fourier transform $\bar{f}$ are connected by the integrals

$$
\begin{aligned}
& \bar{f}(\boldsymbol{p})=(2 \pi)^{-3 / 2} \int \mathrm{e}^{-\mathrm{i} \boldsymbol{p} \cdot \boldsymbol{r}} f(\boldsymbol{r}) \mathrm{d}^{3} \boldsymbol{r}, \\
& f(\boldsymbol{r})=(2 \pi)^{-3 / 2} \int \mathrm{e}^{\mathrm{i} \boldsymbol{r} \cdot \boldsymbol{p}} \bar{f}(\boldsymbol{p}) \mathrm{d}^{3} \boldsymbol{p},
\end{aligned}
$$

\section{B Appendix: Spherical Harmonics}

Normally, the spherical harmonics $Y_{\ell}^{m}(\theta, \phi)$ are introduced as the simultaneous normalized eigenfunctions of the orbital angular momentum operators

$$
\hat{\boldsymbol{L}}^{2}=-\frac{1}{\sin (\theta)} \frac{\partial}{\partial \theta} \sin (\theta) \frac{\partial}{\partial \theta}-\frac{1}{\sin ^{2}(\theta)} \frac{\partial^{2}}{\partial \phi^{2}},
$$

Ernst Joachim Weniger: The Spherical Tensor Gradient Operator 
which is essentially the angular part of the three-dimensional Laplacian in spherical polar coordinates,

$$
\nabla^{2}=\frac{1}{r^{2}} \frac{\partial}{\partial r} r^{2} \frac{\partial}{\partial r}-\frac{\hat{\boldsymbol{L}}^{2}}{r^{2}}
$$

and

$$
\hat{L}_{z}=\mathrm{i} \frac{\partial}{\partial \phi} .
$$

This determines $Y_{\ell}^{m}(\theta, \phi)$ up to an arbitrary phase. If we for instance choose the phase convention of Condon and Shortley [20, Chapter III.4], we obtain the following explicit expression [12, p. 69]:

$$
Y_{\ell}^{m}(\theta, \phi)=\mathrm{i}^{m+|m|}\left[\frac{(2 \ell+1)(\ell-|m|) !}{4 \pi(\ell+|m|) !}\right]^{1 / 2} P_{\ell}^{|m|}(\cos \theta) \mathrm{e}^{\mathrm{i} m \phi} .
$$

Here, $P_{\ell}^{|m|}(\cos \theta)$ is an associated Legendre polynomial [19, p. 155]:

$$
P_{\ell}^{m}(x)=\left(1-x^{2}\right)^{m / 2} \frac{\mathrm{d}^{\ell+m}}{\mathrm{~d} x^{\ell+m}} \frac{\left(x^{2}-1\right)^{\ell}}{2^{\ell} \ell^{\ell}}=\left(1-x^{2}\right)^{m / 2} \frac{\mathrm{d}^{m}}{\mathrm{~d} x^{m}} P_{\ell}(x) .
$$

Alternative phase conventions for the spherical harmonics are discussed in [106, pp. 17 - 22].

The spherical harmonics $Y_{\ell}^{m}(\theta, \phi)$ are often called surface harmonics because the angles $\theta$ and $\phi$ characterize a point $\boldsymbol{r} / r$ on the surface of the three-dimensional unit sphere. In the literature, it is common to introduce the so-called regular and irregular solid harmonics

$$
\begin{aligned}
& \mathcal{Y}_{\ell}^{m}(\boldsymbol{r})=r^{\ell} Y_{\ell}^{m}(\theta, \phi), \\
& \mathcal{Z}_{\ell}^{m}(\boldsymbol{r})=r^{-\ell-1} Y_{\ell}^{m}(\theta, \phi) .
\end{aligned}
$$

Both $\mathcal{Y}_{\ell}^{m}$ and $\mathcal{Z}_{\ell}^{m}$ are defined for arbitrary vectors $r \in \mathbb{R}^{3}$. Moreover, it is easy to show that the regular solid harmonics are for all $\boldsymbol{r} \in \mathbb{R}^{3}$ solutions of the homogeneous three-dimensional Laplace equation

$$
\nabla^{2} f(\boldsymbol{r})=\left[\frac{\partial^{2}}{\partial x^{2}}+\frac{\partial^{2}}{\partial y^{2}}+\frac{\partial^{2}}{\partial z^{2}}\right] f(\boldsymbol{r})=0
$$

whereas the irregular solid harmonics are solutions for all $\boldsymbol{r} \in \mathbb{R}^{3} \backslash\{\mathbf{0}\}$.

In connection with the differential operator $\mathcal{Y}_{\ell}^{m}(\nabla)$, it is more natural to introduce the regular solid harmonics as polynomials satisfying the Laplace

Ernst Joachim Weniger: The Spherical Tensor Gradient Operator 
equation (B.8). Suitable subsets of the polynomials in $x, y$, and $z$ can be characterized by their transformation properties. For example, the homogeneous polynomials $P_{\ell}(x, y, z)$ of degree $\ell$ satisfy

$$
P_{\ell}(\eta x, \eta y, \eta z)=\eta^{\ell} P_{\ell}(x, y, z), \quad \eta \in \mathbb{C}, \quad \ell \in \mathbb{N}_{0} .
$$

A special subset of the homogeneous polynomials of degree $\ell$ are the so-called harmonic polynomials $H_{\ell}(x, y, z)$ of degree $\ell$ that are also solutions of the homogeneous Laplace equation (B.8), i.e., they satisfy

$$
\nabla^{2} H_{\ell}(x, y, z)=0
$$

For a given $\ell \in \mathbb{N}_{0}$ there are exactly $2 \ell+1$ linearly independent harmonic polynomials $H_{\ell}(x, y, z)$ (see for example [43, p. 123] or [74, Appendix H.3]). Accordingly, the regular solid harmonics span the space of harmonic polynomials of degree $\ell$ :

$$
H_{\ell}(x, y, z)=\sum_{m=-\ell}^{\ell} C_{\ell}^{m} \mathcal{Y}_{\ell}^{m}(\boldsymbol{r})
$$

It follows from its definition (B.6) that the regular solid harmonic $\mathcal{Y}_{\ell}^{m}(\boldsymbol{r})$ is a homogeneous harmonic polynomial of degree $\ell$ in the Cartesian components of $\boldsymbol{r}=(x, y, z)$ [12, p. 71]:

$$
\begin{aligned}
\mathcal{Y}_{\ell}^{m}(\boldsymbol{r})= & {\left[\frac{2 \ell+1}{4 \pi}(\ell+m) !(\ell-m) !\right]^{1 / 2} } \\
& \times \sum_{k \geq 0} \frac{(-x-\mathrm{i} y)^{m+k}(x-\mathrm{i} y)^{k} z^{\ell-m-2 k}}{2^{m+2 k}(m+k) ! k !(\ell-m-2 k) !} .
\end{aligned}
$$

\section{Appendix: Gaunt Coefficients}

The so-called Gaunt coefficient [35] is the integral of the product of three spherical harmonics over the surface of the unit sphere in $\mathbb{R}^{3}$ :

$$
\left\langle\ell_{3} m_{3}\left|\ell_{2} m_{2}\right| \ell_{1} m_{1}\right\rangle=\int\left[Y_{\ell_{3}}^{m_{3}}(\Omega)\right]^{*} Y_{\ell_{2}}^{m_{2}}(\Omega) Y_{\ell_{1}}^{m_{1}}(\Omega) \mathrm{d} \Omega
$$

Ernst Joachim Weniger: The Spherical Tensor Gradient Operator 
Gaunt coefficient can be expressed in terms of Clebsch-Gordan coefficients [12. Eq. (3.192)] or $3 \mathrm{jm}$ symbols [19, p. 168], respectively:

$$
\begin{aligned}
\left\langle\ell_{3} m_{3}\left|\ell_{2} m_{2}\right| \ell_{1} m_{1}\right\rangle= & {\left[\frac{\left(2 \ell_{1}+1\right)\left(2 \ell_{2}+1\right)}{4 \pi\left(2 \ell_{3}+1\right)}\right]^{1 / 2} C_{000}^{\ell_{1} \ell_{2} \ell_{3}} C_{m_{1} m_{2} m_{3}}^{\ell_{1} \ell_{2} \ell_{3}} } \\
= & (-1)^{m_{3}}\left[\frac{\left(2 \ell_{1}+1\right)\left(2 \ell_{2}+1\right)\left(2 \ell_{3}+1\right)}{4 \pi}\right]^{1 / 2} \\
& \times\left(\begin{array}{ccc}
\ell_{1} & \ell_{2} & \ell_{3} \\
0 & 0 & 0
\end{array}\right)\left(\begin{array}{ccc}
\ell_{1} & \ell_{2} & \ell_{3} \\
m_{1} & m_{2} & -m_{3}
\end{array}\right) .
\end{aligned}
$$

It follows from the orthonormality of the spherical harmonics that the Gaunt coefficients linearize the product of two spherical harmonics:

$$
Y_{\ell_{1}}^{m_{1}}(\Omega) Y_{\ell_{2}}^{m_{2}}(\Omega)=\sum_{\ell=\ell_{\min }}^{\ell_{\max }}{ }^{(2)}\left\langle\ell m_{1}+m_{2}\left|\ell_{1} m_{1}\right| \ell_{2} m_{2}\right\rangle Y_{\ell}^{m_{1}+m_{2}}(\Omega) .
$$

The symbol $\sum^{(2)}$ indicates that the summation proceeds in steps of two. The summation limits in (C.4), which follow from the selection rules of the $3 \mathrm{jm}$ symbols, are given by [128, Eq. (3.1)]

$$
\begin{aligned}
& \ell_{\max }=\ell_{1}+\ell_{2}, \\
& \ell_{\min }= \begin{cases}\lambda_{\min }, & \text { if } \ell_{\max }+\lambda_{\min } \text { is even, } \\
\lambda_{\min }+1, & \text { if } \ell_{\max }+\lambda_{\min } \text { is odd },\end{cases} \\
& \lambda_{\min }=\max \left(\left|\ell_{1}-\ell_{2}\right|,\left|m_{1}+m_{2}\right|\right) .
\end{aligned}
$$

Gaunt coefficients can be computed according to either (C.2) or (C.3) via the numerous explicit expressions for Clebsch-Gordan coefficients or $3 \mathrm{jm}$ symbols described in the literature. In the case of small angular quantum numbers, this is a satisfactory approach, but in the case of large angular quantum numbers, the computation of individual Clebsch-Gordan coefficients or $3 \mathrm{jm}$ symbols via their explicit expressions becomes computationally costly. In addition, a catastrophic accumulation of rounding errors can easily happen. A much better approach consists in the use of a recurrence formulas for 3jm symbols derived by Schulten and Gordon [97. Although this recursion is neither stable upwards nor downwards, it is nevertheless possible to compute in this way whole strings of $3 \mathrm{jm}$ symbols efficiently and

Ernst Joachim Weniger: The Spherical Tensor Gradient Operator 
reliably even for very large angular momentum quantum numbers 98. In [128], a FORTRAN program is described that computes simultaneously all Gaunt coefficients occurring on the right-hand side of (C.4) with the help of the recurrence formula and the computational algorithm of Schulten and Gordon 97, 98.

It may be of interest for the reader that several articles on Gaunt coefficients have appeared in recent years [45, 137, 138, 139, 100, 68. Then, there are some very recent articles by Dunlad [24, 25, 26, but the objects he considered are not the usual Gaunt coefficients defined in (C.1) but rather generalizations that occur in the context of molecular multicenter integrals of spherical Gaussian functions.

In this article, the following abbreviations will frequently be used:

$$
\begin{aligned}
\Delta \ell & =\left(\ell_{1}+\ell_{2}-\ell\right) / 2, \\
\Delta \ell_{1} & =\left(\ell-\ell_{1}+\ell_{2}\right) / 2, \\
\Delta \ell_{2} & =\left(\ell+\ell_{1}-\ell_{2}\right) / 2, \\
\sigma(\ell) & =\left(\ell_{1}+\ell_{2}+\ell\right) / 2 .
\end{aligned}
$$

If the three orbital angular momentum quantum numbers $\ell_{1}, \ell_{2}$, and $\ell$ satisfy the summation limits (C.5), then these quantities are always positive integers or zero.

\section{References}

[1] F. Arakane and O. Matsuoka, "Recurrence formulas for molecular integrals over Laguerre Gaussian-type functions", Int. J. Quantum Chem. 66, 273 279 (1998).

[2] G. B. Arfken, Mathematical Methods for Physicists, 3rd edition (Academic Press, Orlando, 1985).

[3] J. Avery, Hyperspherical Harmonics - Applications in Quantum Theory (Kluwer, Dordrecht, 1989).

[4] J. Avery, Hyperspherical Harmonics and Generalized Sturmians (Kluwer, Dordrecht, 2000).

[5] G. A. Baker, Jr. and P. Graves-Morris, Padé Approximants, 2nd edition (Cambridge U. P., Cambridge, 1996).

[6] B. F. Bayman, "A generalization of the spherical harmonic gradient formula", J. Math. Phys. 19, $2558-2562$ (1978).

\section{Ernst Joachim Weniger: The Spherical Tensor Gradient Operator}


[7] C. M. Bender and E. J. Weniger, "Numerical evidence that the perturbation expansion for a non-Hermitian $\mathcal{P} \mathcal{T}$-symmetric Hamiltonian is Stieltjes", J. Math. Phys. 42, $2167-2183$ (2001).

[8] L. Berlu and H. Safouhi, "A new algorithm for accurate and fast numerical evaluation of hybrid and three-centre two-electron Coulomb integrals over Slater-type functions", J. Phys. A 36, 11267 - 11283 (2003).

[9] L. Berlu and H. Safouhi, "An extremely efficient and rapid algorithm for numerical evaluation of three-centre nuclear attraction integrals over Slatertype functions", J. Phys. A 36, 11791 - 11805 (2003).

[10] L. Berlu and H. Safouhi, "Multicentre two-electron Coulomb and exchange integrals over Slater functions evaluated using a generalized algorithm based on nonlinear transformations", J. Phys. A 37, 3393 - 3410 (2004).

[11] L. Berlu, H. Safouhi, and P. Hoggan, "Fast and accurate evaluation of threecenter, two-electron Coulomb, hybrid, and three-center nuclear attraction integrals over slater-type orbitals using the $s \bar{D}$ transformation", Int. J. Quantum Chem. 99, 221 - 235 (2004).

[12] L. C. Biedenharn and J. D. Louck, Angular Momentum in Quantum Physics (Addison-Wesley, Reading, Mass., 1981).

[13] E. Bott, M. Methfessel, W. Krabs, and P. C. Schmidt, "Nonsingular Hankel functions as a new basis for electronic structure calculations", J. Math. Phys. 39, 3393 - 3425 (1998).

[14] C. Bracher, T. Kramer, and M. Kleber, "Ballistic matter waves with angular momentum: Exact solutions and applications", Phys. Rev. A 67, 043601-1043601-20 (2003).

[15] P. Bracken and R. J. Bartlett, "Calculation of Gaussian integrals using symbolic manipulation", Int. J. Quantum Chem. 62, 557 - 570 (1997).

[16] L.-Y. Chow Chiu and M. Moharerrzadeh, "Multicenter integrals of spherical Laguerre Gaussian orbitals by generalized spherical gradient operators", J. Chem. Phys. 108, 5230 - 5242 (1998).

[17] L.-Y. Chow Chiu and M. Moharerrzadeh, "Fourier transform of spherical Laguerre Gaussian functions and its application to molecular integrals", Int. J. Quantum Chem. 73, 265 - 273 (1999).

[18] L.-Y. Chow Chiu and M. Moharerrzadeh, "The addition theorem of spherical Laguerre Gaussian functions and its application in molecular integrals", J. Mol. Struc. (Theochem) 536, 263 - 267 (2001).

[19] E. U. Condon and H. Odabaşi, Atomic Structure (Cambridge U. P., Cambridge, 1980).

Ernst Joachim Weniger: The Spherical Tensor Gradient Operator 
[20] E. U. Condon and G. H. Shortley, The Theory of Atomic Spectra (Cambridge U. P., Cambridge, 1970).

[21] P. A. M. Dirac, The Principles of Quantum Mechanics, 4th edition (At the Clarendon Press, Oxford, 1958).

[22] B. I. Dunlap, "Three-center Gaussian-type-orbital integral evaluation using solid spherical harmonics", Phys. Rev. A 42, 1127 - 1137 (1990).

[23] B. I. Dunlap, "Direct quantum chemical integral evaluation", Int. J. Quantum Chem. 81, $373-383$ (2001).

[24] B. I. Dunlap, "Generalized Gaunt coefficients", Phys. Rev. A 66, 032502-1032502-7 (2002).

[25] B. I. Dunlap, "Angular momentum in solid-harmonic-Gaussian integral evaluation", J. Chem. Phys. 118, 1036 - 1043 (2003).

[26] B. I. Dunlap, "Angular momentum in molecular quantum mechanical integral evaluation", Comput. Phys. Commun. 165, 18 - 36 (2005).

[27] G. Fieck, "Racah algebra and Talmi transformations in the theory of multicentre integrals of Gaussian orbitals", J. Phys. B 12, 1063 - 1079 (1979).

[28] G. Fieck, "The multi-centre integrals of derivative, spherical GTOs", Theor. Chim. Acta 54, 323 - 332 (1980).

[29] E. Filter and E. O. Steinborn, "The three-dimensional convolution of reduced Bessel functions and other functions of physical interest", J. Math. Phys. 19, $79-84$ (1978).

[30] E. Filter and E. O. Steinborn, "Extremely compact formulas for molecular one-electron integrals and Coulomb integrals over Slater-type atomic orbitals", Phys. Rev. A 18, 1 - 11 (1978).

[31] E. Filter and E. O. Steinborn, "A matrix representation of the translation operator with respect to a basis of exponentially declining functions", J. Math. Phys. 21, 2725 - 2736 (1980).

[32] A. Fortunelli and V. Carrravetta, "Alternative basis functions for the molecular continuum. II. Integrals with higher-order functions", Phys. Rev. A 45, 4438 - 4451 (1992).

[33] A. Fortunelli and O. Salvetti, "Recurrence relations for the evaluation of electron repulsion integrals over spherical Gaussian functions", Int. J. Quantum Chem. 48, 257 - 265 (1993).

[34] N. Fujimura and O. Matsuoka, "Molecular integrals of Breit interaction over Laguerre Gaussian-type functions", Int. J. Quantum Chem. 42, 751 - 759 (1992).

Ernst Joachim Weniger: The Spherical Tensor Gradient Operator 
[35] J. A. Gaunt, "The triplets of helium", Phil. Trans. Roy. Soc. A 228, 151 196 (1929).

[36] I. M. Gel'fand and G. E. Shilov, Generalized Functions. I. Properties and Operations (Academic Press, New York, 1964).

[37] M. Geller, "Two-electron, one- and two-center integrals", J. Chem. Phys. 39, $853-854$ (1963).

[38] E. Grosswald, Bessel Polynomials (Springer-Verlag, Berlin, 1978).

[39] J. Grotendorst and E. O. Steinborn, "The Fourier transform of a two-center product of exponential-type functions and its efficient evaluation", J. Comput. Phys. 61, $195-217$ (1985).

[40] J. Grotendorst and E. O. Steinborn, "Numerical evaluation of molecular oneand two-center multicenter integrals with exponential-type orbitals via the Fourier-transform method", Phys. Rev. A 38, 3857 - 3876 (1988).

[41] J. Grotendorst, E. J. Weniger, and E. O. Steinborn, "Efficient evaluation of infinite-series representations for overlap, two-center nuclear attraction, and Coulomb integrals using nonlinear convergence accelerators", Phys. Rev. A 33, $3706-3726$ (1986).

[42] E. W. Hobson, "On a theorem in differentiation, and its application to spherical harmonics", Proc. London Math. Soc. 24, 54 - 67 (1892).

[43] E. W. Hobson, The Theory of Spherical and Ellipsoidal Harmonics (Chelsea, New York, 1965). Originally published by Cambridge U. P. (Cambridge, 1931).

[44] H. H. H. Homeier, Integraltransformationsmethode und Quadraturverfahren für Molekülintegrale mit B-Funktionen, Ph.D. thesis, Naturwissenschaftliche Fakultät IV - Chemie und Pharmazie, Universität Regensburg (1990). Published by S. Roderer Verlag, Regensburg.

[45] H. H. H. Homeier and E. O. Steinborn, "Some properties of the coupling coefficients of real spherical harmonics and their relation to Gaunt coefficients", J. Mol. Struc. (Theochem) 368, 31 - 37 (1996).

[46] H. H. H. Homeier, E. J. Weniger, and E. O. Steinborn, "Simplified derivation of a one-range addition theorem of the Yukawa potential", Int. J. Quantum Chem. 44, $405-411$ (1992).

[47] A. Hu, M. Staufer, U. Birkenheuer, V. Igoshine, and N. Rösch, "Analytical evaluation of pseudopotential matrix elements with Gaussian-type solid harmonics of arbitrary angular momentum", J. Quantum Chem. 79, $209-221$ (2000).

Ernst Joachim Weniger: The Spherical Tensor Gradient Operator 
[48] K. Ishida, "Rigorous formula for the fast calculation of the electron repulsion integral over the solid harmonic Gaussian-type orbitals", J. Chem. Phys. 109, 881 - 890 (1998).

[49] K. Ishida, "Rigorous and rapid calculation of the electron repulsion integral over the uncontracted solid harmonic Gaussian-type orbitals", J. Chem. Phys. 111, 4913 - 4922 (1999).

[50] K. Ishida, "Rigorous algorithm for the electron repulsion integral over the generally contracted solid harmonic Gaussian-type orbitals", J. Chem. Phys. 113, $7818-7829$ (2000).

[51] K. Ishida, "Accompanying coordinate expansion formulas derived with the solid harmonic gradient", J. Comput. Chem. 23, 378 - 393 (2002).

[52] K. Ishida, "Molecular integrals over the gauge-including atomic orbitals. II. the Breit-Pauli interaction", J. Comput. Chem. 24, 1874 - 1890 (2003).

[53] J. D. Jackson, Classical Electrodynamics, 2nd edition (Wiley, New York, 1975).

[54] M. N. Jones, Spherical Harmonics and Tensors for Classical Field Theory (Wiley, New York, 1985).

[55] B. R. Judd, Angular Momentum Theory for Diatomic Molecules (Academic Press, New York, 1975).

[56] M. Kline, Mathematical Thought from Ancient to Modern Times (Oxford U. P., Oxford, 1972).

[57] K. Knopp, Theorie und Anwendung der unendlichen Reihen (Springer-Verlag, Berlin, 1964).

[58] J. Kuang and C. D. Lin, "Molecular integrals over spherical Gaussian-type orbitals. I", J. Phys. B 30, 2529 - 2548 (1997).

[59] J. Kuang and C. D. Lin, "Molecular integrals over spherical Gaussian-type orbitals. II. Modified with plane wave factors", J. Phys. B 30, $2549-2567$ (1997).

[60] D. Levin and A. Sidi, "Two new classes of nonlinear transformations for accelerating the convergence of infinite integrals and series", Appl. Math. Comput. 9, $175-215$ (1981).

[61] W. Magnus, F. Oberhettinger, and R. P. Soni, Formulas and Theorems for the Special Functions of Mathematical Physics (Springer-Verlag, New York, 1966).

[62] D. K. Maretis, "Talmi transformation and the multicenter integrals of harmonic oscillator functions", J. Chem. Phys. 71, 917 - 921 (1979).

Ernst Joachim Weniger: The Spherical Tensor Gradient Operator 
[63] O. Matsuoka, "Molecular integrals over spherical Laguerre Gaussian-type functions", J. Chem. Phys. 92, $4364-4371$ (1992).

[64] O. Matsuoka, "Molecular integrals over Laguerre Gaussian-type functions of real spherical harmonics", Can. J. Chem. 70, 388 - 392 (1992).

[65] O. Matsuoka, "Molecular integrals over real solid spherical Gaussian-type functions", J. Chem. Phys. 108, 1063 - 1067 (1998).

[66] O. Matsuoka, "Simple derivations of Talmi coefficients for solid spherical Gaussian-type functions", J. Mol. Struc. (Theochem) 451, 35 - 39 (1998).

[67] O. Matsuoka, "A simple calculation of Talmi coefficients of Laguerre Gaussians", Mol. Phys. 101, 33 - 36 (2003).

[68] H. A. Mavromatis and R. S. Alassar, "A generalized formula for the integral of three associated Legendre polynomials", Appl. Math. Lett. 12, 101 - 105 (1999).

[69] M. Methfessel, M. van Schilfgaarde, and R. A. Casali, "A full-potential LMTO method based on smooth Hankel functions", in H. Dreyssé (Editor), Electronic Structure and Physical Properties of Solids. The Use of the LMTO Method, volume 535 of Lecture Notes in Physics, 114 - 147 (Springer-Verlag, Berlin, 1999).

[70] A. W. Niukkanen, "A new class of gradient formulas in the angular momentum theory", J. Math. Phys. 24, 1989 - 1991 (1983).

[71] A. W. Niukkanen, "Generating differential operators for the basis functions in the variational LCAO-type methods", J. Math. Phys. 25, 698 - 705 (1984).

[72] A. W. Niukkanen, "Fourier transforms of atomic orbitals. I. Reduction to four-dimensional harmonics and quadratic transformations", Int. J. Quantum Chem. 25, 941 - 955 (1984).

[73] A. W. Niukkanen, "Operator Racah algebra and Laplace-type expansions of irreducible spherical tensors", J. Math. Phys. 26, 1540 - 1546 (1985).

[74] J.-M. Normand, A Lie Group: Rotations in Quantum Mechanics (NorthHolland, Amsterdam, 1980).

[75] B. K. Novosadov, "Hydrogen-like atomic orbitals: Addition and expansion theorems, integrals", Int. J. Quantum Chem. 24, 1 - 18. (1983).

[76] B. K. Novosadov, "Appendix: Methods for computing matrix elements in the theory of electronic spectra", in I. A. Gribov and W. J. Orville-Thomas (Editors), Theory and Methods of Calculation of Molecular Spectra, 596-625 (Wiley, Chichester, 1988).

\section{Ernst Joachim Weniger: The Spherical Tensor Gradient Operator}


[77] B. K. Novosadov, "Method of calculating two-center two-particle matrix elements from a screened Coulomb potential in an exponential AO basis", J. Struc. Chem. 42, 355 - 359 (2001).

[78] B. K. Novosadov, "Calculation of 4-center Coulomb repulsion matrix elements in a basis of exponential type spherical AO using 9-dimensional polyspherical harmonics", J. Struc. Chem. 43, 383 - 389. (2002).

[79] B. K. Novosadov, "Calculation of 3-center one-electron integrals $\left\langle A B \mid r_{C}^{-1}\right\rangle$ and Coulomb repulsion matrix elements $\left\langle A B \mid C C^{\prime}\right\rangle$ in an exponential type spherical AO basis", J. Struc. Chem. 43, 390 - 400 (2002).

[80] B. K. Novosadov, "Besselian: Calculation of multicenter matrix elements", J. Mol. Struc. (Theochem) 664 - 665, 55 - 81 (2003).

[81] H. Padé, "Sur la représentation approachée d'une fonction par des fractions rationelles", Ann. Sci. Ec. Norm. Sup. 9, 1 - 93 (1892).

[82] F. P. Prosser and C. H. Blanchard, "On the evaluation of two-center integrals", J. Chem. Phys. 36, 1112 (1962).

[83] M. A. Rashid, "Simple expressions for radial functions appearing in the expansions of $\mathcal{Y}_{\ell_{1}}^{m_{1}}(\nabla) F_{\ell_{2}}^{m_{2}}(\boldsymbol{r})$ and $\nabla^{2 n} \mathcal{Y}_{\ell_{1}}^{m_{1}}(\nabla) F_{\ell_{2}}^{m_{2}}(\boldsymbol{r}) "$, J. Math. Phys. 27, 549 - 551 (1986).

[84] M. Reed and B. Simon, Methods of Modern Mathematical Physics II: Fourier Analysis, Self-Adjointness (Academic Press, New York, 1975).

[85] E. G. P. Rowe, "Spherical delta functions and multipole expansions", J. Math. Phys. 19, 1962 - 1968 (1978).

[86] H. Safouhi, "The $H D$ and $H \bar{D}$ methods for accelerating the convergence of three-center nuclear attraction and four-center two-electron Coulomb integrals over $B$ functions and their convergence properties", J. Comput. Phys. 165, $473-495$ (2000).

[87] H. Safouhi, "An extremely efficient approach for accurate and rapid evaluation of three-centre two-electron Coulomb and hybrid integrals over $B$ functions", J. Phys. A 34, 881 - 902 (2001).

[88] H. Safouhi, "The properties of sine, spherical Bessel and reduced Bessel functions for improving convergence of semi-infinite very oscillatory integrals: The evaluation of three-centre nuclear attraction integrals over $B$ functions", J. Phys. A 34, $2801-2818$ (2001).

[89] H. Safouhi, "Numerical evaluation of three-center two-electron Coulomb and hybrid integrals over $B$ functions using the $H D$ and $H \bar{D}$ methods and convergence properties", J. Math. Chem. 29, 213 - 232 (2001).

Ernst Joachim Weniger: The Spherical Tensor Gradient Operator 
[90] H. Safouhi, "Efficient and rapid numerical evaluation of the two-electron, fourcenter Coulomb integrals using nonlinear transformations and useful properties of sine and Bessel functions", J. Comput. Phys. 176, 1 - 19 (2002).

[91] H. Safouhi, "Convergence properties of the $S \bar{D}$ transformation and a fast and accurate numerical evaluation of molecular integrals", J. Phys. A 35, 9685 9698 (2002).

[92] H. Safouhi and P. E. Hoggan, "Recent progress in the accurate and rapid evaluation of all Coulomb integrals over Slater-type orbitals", Int. J. Quantum Chem. 84, 580 - 591 (2001).

[93] H. Safouhi and P. E. Hoggan, "Three-center nuclear attraction, three-center two-electron Coulomb and hybrid integrals over $B$ functions evaluated using the nonlinear $S \bar{D}$ transformation", Int. J. Quantum Chem. 90, $119-135$ (2002).

[94] H. Safouhi and P. E. Hoggan, "New methods for accelerating the convergence of molecular electronic integrals over exponential type orbitals", Mol. Phys. 101, $19-31$ (2003).

[95] F. D. Santos, "Finite range approximation in direct transfer reactions", Nucl. Phys. A 212, $341-364$ (1973).

[96] V. R. Saunders, "Molecular integrals for Gaussian type functions", in G. Diercksen and S. Wilson (Editors), Methods in Computational Molecular Physics, 1 - 36 (Reidel, Dordrecht, 1983).

[97] K. Schulten and R. G. Gordon, "Exact recursive evaluation of $3 j$ - and $6 j$ coefficients for quantum-mechanical coupling of angular momenta", J. Math. Phys. 16, 1961 - 1970 (1975).

[98] K. Schulten and R. G. Gordon, "Recursive evaluation of $3 j$ and $6 j$ coefficients", Comput. Phys. Commun. 11, 269 - 278 (1976).

[99] L. Schwartz, Théorie des Distributions, 2nd edition (Hermann, Paris, 1966).

[100] D. Sébilleau, "On the computation of the integrated product of three spherical harmonics", J. Phys. A 31, 7157 - 7168 (1998).

[101] I. Shavitt, "The Gaussian function in calculations of statistical mechanics and quantum mechanics", in B. Alder, S. Fernbach, and M. Rotenberg (Editors), Methods in Computational Physics Vol. 2. Quantum Mechanics, 1 - 45 (Academic Press, New York, 1963).

[102] A. Sidi, "Extrapolation methods for oscillatory infinite integrals", J. Inst. Math. Applic. 26, 9 - 17 (1980).

Ernst Joachim Weniger: The Spherical Tensor Gradient Operator 
[103] E. O. Steinborn and E. Filter, "Translations of fields represented by sphericalharmonic expansions for molecular calculations. III. Translations of reduced Bessel functions, Slater-type s-orbitals, and other functions", Theor. Chim. Acta 38, $273-281$ (1975).

[104] E. O. Steinborn, H. H. H. Homeier, I. Ema, R. López, and G. Ramírez, "Molecular calculations with $B$ functions", Int. J. Quantum Chem. 76, 244 251 (2000).

[105] E. O. Steinborn, H. H. H. Homeier, J. Fernández Rico, I. Ema, R. López, and G. Ramírez, "An improved program for molecular calculations with $B$ functions", J. Mol. Struc. (Theochem) 490, 201 - 217 (1999).

[106] E. O. Steinborn and K. Ruedenberg, "Rotation and translation of regular and irregular solid spherical harmonics", Adv. Quantum Chem. 7, 1 - 81 (1973).

[107] E. O. Steinborn and E. J. Weniger, "Advantages of reduced Bessel functions as atomic orbitals: An application to $\mathrm{H}_{2}^{+}$", Int. J. Quantum Chem. Symp. 11, $509-516(1977)$.

[108] E. O. Steinborn and E. J. Weniger, "Sequence transformations for the efficient evaluation of infinite series representations of some molecular integrals with exponentially decaying basis functions", J. Mol. Struc. (Theochem) 210, 71 -78 (1990).

[109] E. O. Steinborn and E. J. Weniger, "Nuclear attraction and electron interaction integrals of exponentially decaying functions and the Poisson equation", Theor. Chim. Acta 83, 105 - 121 (1992).

[110] O. Steinborn, "Poly-polar expansions for the regular and irregular spherical harmonics in molecules", Chem. Phys. Lett. 3, 671 - 675 (1969).

[111] A. J. Stone, The Theory of Intermolecular Forces (Clarendon Press, Oxford, 1996).

[112] S. N. Stuart, "Non-classical integrals of Bessel functions", J. Austral. Math. Soc. B 22, $368-378$ (1981).

[113] H. Tai, "Generalized method for solving multi-center molecular integrals over Slater type orbitals", Chin. J. Phys. 32, 847 - 862 (1994).

[114] D. A. Varshalovich, A. N. Moskalev, and V. K. Khersonskii, Quantum Theory of Angular Momentum (World Scientific, Singapore, 1988).

[115] G. N. Watson, A Treatise on the Theory of Bessel Functions (Cambridge U. P., Cambridge, 1966).

[116] E. J. Weniger, Untersuchung der Verwendbarkeit reduzierter Besselfunktionen als Basissatz für ab initio Rechnungen an Molekülen. Vergleichende Rechnungen am Beispiel des $\mathrm{H}_{2}^{+}$, Diplomarbeit, Fachbereich Chemie und Pharmazie, Universität Regensburg (1977).

Ernst Joachim Weniger: The Spherical Tensor Gradient Operator 
[117] E. J. Weniger, Reduzierte Bessel-Funktionen als LCAO-Basissatz: Analytische und numerische Untersuchungen, Ph.D. thesis, Fachbereich Chemie und Pharmazie, Universität Regensburg (1982). A short abstract of this thesis was published in Zentralblatt für Mathematik 523, 444 (1984), abstract no. 65015 .

[118] E. J. Weniger, "Weakly convergent expansions of a plane wave and their use in Fourier integrals", J. Math. Phys. 26, 276 - 291 (1985).

[119] E. J. Weniger, "Nonlinear sequence transformations for the acceleration of convergence and the summation of divergent series", Comput. Phys. Rep. 10, 189 - 371 (1989). Los Alamos Preprint math-ph/0306302 (http://arXiv.org).

[120] E. J. Weniger, "Addition theorems as three-dimensional Taylor expansions", Int. J. Quantum Chem. 76, 280 - 285 (2000).

[121] E. J. Weniger, "Prediction properties of Aitken's iterated $\Delta^{2}$ process, of Wynn's epsilon algorithm, and of Brezinski's iterated theta algorithm", J. Comput. Appl. Math. 122, 329 - 356 (2000). Reprinted in C. Brezinski (Editor), Numerical Analysis 2000, Vol. 2: Interpolation and Extrapolation, 329 - 356 (Elsevier, Amsterdam, 2000).

[122] E. J. Weniger, "Addition theorems as three-dimensional Taylor expansions. II. $B$ functions and other exponentially decaying functions", Int. J. Quantum Chem. 90, 92 - 104 (2002).

[123] E. J. Weniger, "A rational approximant for the digamma function", Numer. Algor. 33, 499 - 507 (2003).

[124] E. J. Weniger, "Mathematical properties of a new Levin-type sequence transformation introduced by Č́žzek, Zamastil, and Skála. I. Algebraic theory", J. Math. Phys. 45, 1209 - 1246 (2004).

[125] E. J. Weniger, J. Grotendorst, and E. O. Steinborn, "Some applications of nonlinear convergence accelerators", Int. J. Quantum Chem. Symp. 19, 181 - 191 (1986).

[126] E. J. Weniger, J. Grotendorst, and E. O. Steinborn, "Unified analytical treatment of overlap, two-center nuclear attraction, and Coulomb integrals of $B$ functions via the Fourier transform method", Phys. Rev. A 33, 3688 - 3705 (1986).

[127] E. J. Weniger and B. Kirtman, "Extrapolation methods for improving the convergence of oligomer calculations to the infinite chain limit of quasionedimensional stereoregular polymers", Comput. Math. Applic. 45, 189 215 (2003).

Ernst Joachim Weniger: The Spherical Tensor Gradient Operator 
[128] E. J. Weniger and E. O. Steinborn, "Programs for the coupling of spherical harmonics", Comput. Phys. Commun. 25, 149 - 157 (1982).

[129] E. J. Weniger and E. O. Steinborn, "The Fourier transforms of some exponential-type functions and their relevance to multicenter problems", J. Chem. Phys. 78, $6121-6132$ (1983).

[130] E. J. Weniger and E. O. Steinborn, "Numerical properties of the convolution theorems of $B$ functions", Phys. Rev. A 28, 2026 - 2041 (1983).

[131] E. J. Weniger and E. O. Steinborn, "New representations for the spherical tensor gradient and the spherical delta function", J. Math. Phys. 24, 2553 2563 (1983).

[132] E. J. Weniger and E. O. Steinborn, "Comment on "Hydrogenlike orbitals referred to another coordinate"”, Phys. Rev. A 29, 2268 - 2271 (1984).

[133] E. J. Weniger and E. O. Steinborn, "A simple derivation of the addition theorems of the irregular solid harmonics, the Helmholtz harmonics, and the modified Helmholtz harmonics", J. Math. Phys. 26, 664 - 670 (1985).

[134] E. J. Weniger and E. O. Steinborn, "Comment on "Molecular overlap integrals with exponential-type integrals"”, J. Chem. Phys. 87, 3709 - 3711 (1987).

[135] E. J. Weniger and E. O. Steinborn, "Overlap integrals of $B$ functions. A numerical study of infinite series representations and integral representations", Theor. Chim. Acta 73, 323 - 336 (1988).

[136] E. J. Weniger and E. O. Steinborn, "Addition theorems for $B$ functions and other exponentially declining functions", J. Math. Phys. 30, $774-784$ (1989).

[137] Y.-L. Xu, "Fast evaluation of Gaunt coefficients", Math. Comput. 65, 1601 $1612(1996)$.

[138] Y.-L. Xu, "Fast evaluation of Gaunt coefficients: Recursive approach", J. Comput. Appl. Math. 85, 53 - 65 (1997).

[139] Y.-L. Xu, "Efficient evaluation of vector translation coefficients in multiparticle light-scattering theories", J. Comput. Phys. 139, 137 - 165 (1998).

[140] H. Yukawa, "On the interaction of elementary particles. I.", Proc. Phys.-Math. Soc. Japan 17, $48-57$ (1935).

Ernst Joachim Weniger: The Spherical Tensor Gradient Operator 